\title{
Astrocytes in the Ventrolateral Preoptic Area Promote Sleep
}

\author{
Jae-Hong Kim, ${ }^{1}$ In-Sun Choi, ${ }^{2}$ Ji-Young Jeong, ${ }^{1}$ Il-Sung Jang, ${ }^{2,3}{ }^{\circledR}$ Maan-Gee Lee, ${ }^{1,3}$ and ${ }^{\circledR}$ Kyoungho Suk $^{1,3}$ \\ ${ }^{1}$ Department of Pharmacology, School of Medicine, Kyungpook National University, Daegu 41944, Republic of Korea, ${ }^{2}$ Department of \\ Pharmacology, School of Dentistry, Kyungpook National University, Daegu 41940, Republic of Korea, and ${ }^{3}$ Brain Science and Engineering Institute, \\ Kyungpook National University, Daegu 41566, Republic of Korea
}

\begin{abstract}
Although ventrolateral preoptic (VLPO) nucleus is regarded as a center for sleep promotion, the exact mechanisms underlying the sleep regulation are unknown. Here, we used optogenetic tools to identify the key roles of VLPO astrocytes in sleep promotion. Optogenetic stimulation of VLPO astrocytes increased sleep duration in the active phase in naturally sleep-waking adult male rats $(n=6)$; it also increased the extracellular ATP concentration $(n=3)$ and c-Fos expression $(n=3-4)$ in neurons within the VLPO. In vivo microdialysis analyses revealed an increase in the activity of VLPO astrocytes and ATP levels during sleep states $(n=4)$. Moreover, metabolic inhibition of VLPO astrocytes reduced ATP levels $(n=4)$ and diminished sleep duration $(n=4)$. We further show that tissue-nonspecific alkaline phosphatase (TNAP), an ATP-degrading enzyme, plays a key role in mediating the somnogenic effects of ATP released from astrocytes $(n=5)$. An appropriate sample size for all experiments was based on statistical power calculations. Our results, taken together, indicate that astrocyte-derived ATP may be hydrolyzed into adenosine by TNAP, which may in turn act on VLPO neurons to promote sleep.
\end{abstract}

Key words: astrocyte; gliotransmitters; optogenetics; sleep; ventrolateral preoptic area

Significance Statement

Glia have recently been at the forefront of neuroscience research. Emerging evidence illustrates that astrocytes, the most abundant glial cell type, are the functional determinants for fates of neurons and other glial cells in the central nervous system. In this study, we newly identified the pivotal role of hypothalamic ventrolateral preoptic (VLPO) astrocytes in the sleep regulation, and provide novel insights into the mechanisms underlying the astrocyte-mediated sleep regulation.

\section{Introduction}

The maintenance of neural activity involves many energy-consuming processes; consequently, sleep is required to restore energy (Benington and Heller, 1995). Sleep, which is also associated with a number of brain functions including memory consolidation and synaptic plasticity (Bezzi and Volterra, 2001), is regulated by two processes: (1) the circadian rhythm and (2) sleep propensity (also known as pressure), which depends on prior brain activity during waking (Tobler and Borbély, 1990; Borbély et al., 2016). In this regard, sleep promotion based on sleep need requires simultaneous "sleep-on" and "wake-off" signals; therefore, neural circuits

Received June 10, 2020; revised 0ct. 5, 2020; accepted 0ct. 13, 2020.

Author contributions: J.-H.K., I.-S.C. designed \& carried out experiments, collected \& interpreted data, prepared illustrations, reviewed \& edited the manuscript; J.-Y.J. designed \& carried out experiments, collected \& interpreted data; I.-S.J., M.-G.L., K.S. conceptualized \& designed experiments, supervised the study, interpreted data, obtained funding, and wrote the manuscript. All authors read and approved the final version of the manuscript

This work was supported by the Korean Government (MSIP) NRF Grants 2012R1A2A2A02046812 and 2016M3C7A1904148. We thank Dr. B. E. Jones for her valuable comments to this paper.

The authors declare no competing financial interests.

I.-S.C.'spresent address: Korea Brain Research Institute, Daegu 41068, Republic of Korea.

Correspondence should be addressed to Kyoungho Suk at ksuk@knu.ac.kr or Maan-Gee Lee at mglee@ knu.ac.kr.

https://doi.org/10.1523/JNEUROSCI.1486-20.2020

Copyright $(2020$ the authors that facilitate a reciprocal inhibition between sleep and arousal centers play a pivotal role in sleep promotion (Sherin et al., 1998; Chou et al., 2002; Saper et al., 2010). In particular, the ventrolateral preoptic (VLPO) area, which modulates several arousal centers within the brain by GABAergic inhibitory projections (Sherin et al., 1998; Steininger et al., 2001; Saper et al., 2010), is one of the key nuclei that promote sleep (Gallopin et al., 2000; Saper et al., 2010). Indeed, lesions in the VLPO area cause insomnia and impairment of NREM sleep (Lu et al., 2000). In a group of neurons within the VLPO area, the expression of c-Fos protein has been shown to specifically increase during sleep but not during wakefulness (Sherin et al., 1996).

A growing body of evidence suggests that astrocytes interact with adjacent neurons through the release of distinct neuroactive substances, known as gliotransmitters (which include glutamate, ATP, D-serine, and GABA), in response to adequate stimulation such as neuronal activity (Santello et al., 2012; Araque et al., 2014). Consequently, astrocytes can modulate several physiological brain functions, including synaptic transmission, breathing, perception, learning, and memory (Ben Achour and Pascual, 2012; Frank, 2013; Araque et al., 2014; Perea et al., 2014). Astrocytes also play an important role in sleep homeostasis and in the cognitive decline associated with sleep loss (Halassa et al., 2009). Such global changes in sleep homeostasis can be mediated by astrocytic release of ATP and the subsequent hydrolysis of 
ATP to adenosine, and these changes depend on adenosine $A_{1}$ receptors (Halassa et al., 2009). Also, stimulation of local astrocytes in the area responsible for sleep-wake regulation, such as posterior hypothalamus, increases sleep (Pelluru et al., 2016). Adenosine is therefore regarded as an endogenous sleep-promoting substance (Porkka-Heiskanen et al., 1997; Porkka-Heiskanen and Kalinchuk, 2011).

The somnogenic effects of exogenously applied adenosine on several brain regions, including the VLPO area, have previously been documented (Ticho and Radulovacki, 1991; Huston et al., 1996; Porkka-Heiskanen et al., 1997; Gallopin et al., 2005; Methippara et al., 2005; Zhang et al., 2013; Liu et al., 2017). However, the mechanisms underlying the sleep-regulating effect of local astrocytes and gliotransmitters derived from them within the VLPO area have yet to be established. In the present study, therefore, we used optogenetic tools (Figueiredo et al., 2011) to investigate the functional roles of local astrocytes within the VLPO sleep center and to elucidate the possible mechanisms underlying the neuron-astrocyte interaction.

\section{Materials and Methods}

All experiments complied with the guiding principles for the care and use of animals approved by the National Institutes of Health Guide for the Care and Use of Laboratory Animals, and every effort was made to minimize both the number of animals used and their suffering. All animals were housed individually in Plexiglas cages $(28 \times 42 \times 18 \mathrm{~cm})$ in a controlled environment at $21-24^{\circ} \mathrm{C}$ and with a $12 / 12 \mathrm{~h}$ light/dark cycle (lights on from 7 A.M. to 7 P.M. for the rats injected with the adenoviral vector or lights on from 6 A.M. to 6 P.M. for the rats injected with the adeno-associated viral vector). They were fed a standard commercial food, and tap water was made available ad libitum, except on the days of the recordings. Adult male Sprague Dawley (SD) rats (275-300 g, 812 weeks old) were used for all behavioral studies and microdialysis experiments, except where indicated. There was no discrepancy between the number of animals at the beginning and at the end of the study. All relevant data shown in this study are available from the authors.

\section{Molecular, cellular, and histologic studies \\ Viral gene transfer in vivo}

Adult rats were anesthetized with 2-4\% isoflurane (Baxter) and placed on a stereotaxic frame. Ventral regions of the VLPO area were targeted unilaterally or bilaterally for microinjections of adenoviral vectors (AdChR2-Kat1.3; generously provided by Kasparov at University of Bristol, Bristol, UK) using the following coordinates: antero-posterior (A) -0.4 $\mathrm{mm}$, lateral (L) $\pm 1.1 \mathrm{~mm}$ from bregma, and ventral $(\mathrm{V})-8.5 \mathrm{~mm}$ from dura. Alternatively, adeno-associated viral vectors (AAV-ChR2-eYFP; University of North Carolina Gene Therapy Program Vector Core) were similarly injected into the same VLPO region. After microinjection, the wound was sutured. Immediate postoperative care was given, and animals were left to recover for $7 \mathrm{~d}$ before the experiments to ensure a high level of transgene expression. In all behavioral experiments using viral vectors (Ad-ChR2-Kat1.3, Ad-eGFP, AAV-ChR2-eYFP, and AAV-eYFP), the expression of the relevant proteins within the VLPO region was verified as fluorescent signals at the end of each experiment. Data from behavioral experiments for which fluorescent signals were not detected within the VLPO region were discarded. A combination of Ad-ChR2-Kat1.3 and Ad-eGFP was successfully used for optogenetic stimulation of spinal astrocytes in one of our previous studies (Nam et al., 2016).

\section{Confocal imaging of $\mathrm{Ca}^{2+}$ responses in cultured astrocytes}

After infection with Ad-ChR2-Kat1.3 or AAV-ChR2-eYFP, primary astrocytes were loaded with Fluo- 4 or rhodamine 2. Images were then obtained using a confocal microscope with a water-immersion objective $(40 \times)$. A 488-nm Argon laser was used to activate channelrhodopsin-2 (ChR2) and excite Fluo-4, and measurements were taken using a bandpass filter $(505-550 \mathrm{~nm})$. The illumination intensity was kept to a minimum by limiting it to $0.5-0.7 \%$ of laser output.

\section{Microdialysis}

Adult rats were maintained under isoflurane anesthesia and mounted onto a stereotaxic frame. Two guide cannulae, one for optical stimulation and the other for microdialysis, were implanted into the VLPO region. The tips of the guide cannulae were lowered to $1.0 \mathrm{~mm}$ above the target: A $-0.4 \mathrm{~mm}, \mathrm{~L} \pm 1.1 \mathrm{~mm}$ from the bregma, and $\mathrm{V}-8.5 \mathrm{~mm}$ from the dura. These were fixed to the skull with three anchor screws using dental cement. The cannula for optic stimulation was inserted obliquely (at an angle of $20^{\circ}$ ); the microdialysis probe cannula was inserted perpendicularly into the VLPO region. Following the cannula implantation, the rats were housed individually and allowed to recover for $7 \mathrm{~d}$. The microdialysis probe (CMA Microdialysis $\mathrm{AB}$ ) was inserted into the VLPO region through the guide cannula $24 \mathrm{~h}$ before the start of the microdialysis. The probe was connected to a microperfusion pump with polyethylene tubing and perfused with artificial CSF (ACSF) at a flow rate of $0.5 \mu \mathrm{l} / \mathrm{min}$. Extracellular fluid (ECF) from the outlet end of the tube was collected in plastic vials kept on ice. Samples were collected for $120 \mathrm{~min}$ with 60-min intervals. Collected samples were immediately frozen at $-80^{\circ} \mathrm{C}$ until analysis of ATP, adenosine, other gliotransmitters, and cytokines. For some experiments, ECF was collected after administration of a metabolic inhibitor of astrocytes [L- $\alpha$-aminoadipate (L- $\alpha$-AA), $10 \mathrm{~nm}$; Sigma-Aldrich] or 2,5-dimethoxy-N-(quinolin-3-yl)benzenesulfonamide [tissue-nonspecific alkaline phosphatase (TNAP) inhibitor (TNAP-I); Merck Millipore]. Cresyl violet staining of brain sections (sagittal and coronal) was done for histologic verification of probe placement.

\section{Immunohistochemistry}

Animals were anesthetized with diethyl ether and transcardially perfused first with saline and then with $4 \%$ paraformaldehyde diluted in $100 \mathrm{~mm}$ PBS. The brains were fixed in $4 \%$ paraformaldehyde for $3 \mathrm{~d}$ before being cryo-protected using a $30 \%$ sucrose solution for a further $3 \mathrm{~d}$. The fixed brains were embedded in OCT compound (Tissue-Tek; Sakura Finetek) and then sectioned to a thickness of $20-25 \mu \mathrm{m}$. For the double or triple immunofluorescence analysis, tissue sections were incubated with mouse anti-GFAP antibody (1:500 dilution; BD Biosciences, catalog \#556330; Jha et al., 2015), mouse anti-GAD67 (1:500 dilution; Millipore, catalog \#MAB5406; Guo et al., 2014), mouse anti-c-Fos (1:500 dilution; Santa Cruz Biotech, catalog \#sc-8047; Grafe et al., 2014), rabbit anti-GFAP (1:1000 dilution; Dako, catalog \#Z0334; Van Poucke et al., 2016), rabbit anti-Iba-1 (1:1000 dilution; Wako, catalog \#019-19741; Choudhury et al., 2016), and mouse anti-NeuN (1:500 dilution; Millipore, catalog \#MAB377; Guenthner et al., 2013). Sections were visualized via incubation with Cy3-conjugated, Cy5-conjugated, and FITC-conjugated antimouse, anti-rabbit, or anti-goat IgG antibodies (The Jackson Laboratory; Cy3-mouse, catalog \#715-165-151; Cy3-rabbit, catalog \#711-165-152; Cy3-goat, catalog \#705-165-147; Cy5-rabbit, catalog \#711-175-152; FITC-mouse, catalog \#715-095-151; FITC-rabbit, catalog \#711-096152; FITC-goat, catalog \#705-095-147) and examined under fluorescence or confocal microscopes. Fluorescence intensities were quantified using ImageJ software version 1.44 (National Institutes of Health). For quantitative analysis, images were obtained from three non-overlapping fields chosen randomly within the VLPO area.

\section{Bioluminescence assay}

The extracellular ATP content of the samples was determined using an ATP bioluminescence assay kit (Sigma) and a Synergy four MultiDetection Microplate Reader (BioTek Instruments Inc.). Undiluted samples $(100 \mu \mathrm{l})$ of the culture medium from primary astrocytes or diluted samples of dialysate $(25 \mu \mathrm{l}$ of the dialysate from the buffer-perfused brain tissue mixed with $75 \mu$ l of ultrapure water) were pipetted into a 96-well microplate for ATP analysis. ATP assay mix solution $(100 \mu \mathrm{l})$ was added to each well and the luminescence signal was assessed kinetically within $1 \mathrm{~min}$.

\section{Analysis of gliotransmitters using an amino acid auto-analyzer}

Dialysate samples $(50 \mu \mathrm{l})$, which were hydrolyzed with $6 \mathrm{~N} \mathrm{HCl}$, were injected into an amino acid auto-analyzer (L-8900, Hitachi). Free amino acids were then separated on an ion exchange column (2622 SC PF, Hitachi) at $50^{\circ} \mathrm{C}$ and eluted with a buffer set (PF-1, PF-2, PF-3, PF-4, PF-6, PF-RG, R-3, and C-1) as a mobile phase. Subsequently, the eluted 
free amino acids were reacted with ninhydrin solution (Wako) at $135^{\circ} \mathrm{C}$ in a post-column. Finally, the detected ninhydrin-labeled free amino acids, which were measured at wavelengths of 570 and $440 \mathrm{~nm}$, were quantified. A mixture of type ANII and type B (Wako) was used as an internal standard for free amino acid quantification.

\section{Cytokine ELISA}

Levels of TNF- $\alpha$ and IL- $1 \beta$ in the brain dialysate samples were measured using specific ELISA assays (R\&D Systems). The assays were performed in 96-well plates using ECF (1:50 dilution) as per the manufacturer's instructions. For standard, recombinant TNF- $\alpha$ or IL$1 \beta$ protein was used at concentrations ranging from 10 to $2500 \mathrm{pg} / \mathrm{ml}$. Recombinant TNF- $\alpha$ and IL- $1 \beta$ protein levels were normalized to the total protein content of the ECF samples. All measurements were obtained from duplicated assays.

\section{Primary cultures of astrocytes}

Neonatal astrocyte cultures were prepared from mixed glial cultures as previously described but with minor modifications. In brief, the whole brain of SD rats ( $2-3 \mathrm{~d}$ old, both sexes) was chopped and mechanically disrupted using a nylon mesh. The cells obtained were seeded in culture flasks and grown at $37^{\circ} \mathrm{C}$ in a $5 \% \mathrm{CO}_{2}$ atmosphere in DMEM supplemented with $10 \%$ fetal bovine serum (FBS) and $100 \mathrm{U} / \mathrm{ml}$ penicillinstreptomycin (Invitrogen-BRL). Culture media were changed initially after $5 \mathrm{~d}$ and then every $3 \mathrm{~d}$, and cells were used after being cultured for 14-21 d. Primary astrocytes were obtained by shaking mixed glial cultures at $250 \mathrm{rpm}$ overnight. Culture media were discarded, and then astrocytes were dissociated using trypsin-EDTA (Invitrogen-BRL) before being collected following centrifugation at $1200 \mathrm{rpm}$ for $10 \mathrm{~min}$. Finally, primary astrocyte cultures were grown and maintained in DMEM supplemented with $10 \% \mathrm{FBS}$ and penicillin-streptomycin at $37^{\circ} \mathrm{C}$ in a humidified $5 \% \mathrm{CO}_{2}$ incubator.

\section{In vivo sleep recordings}

Surgery and microinjection of viral vectors

Adult rats were anesthetized with an intraperitoneal injection of a $2 \mathrm{ml} /$ $\mathrm{kg}$ cocktail of ketamine hydrochloride $(72.6 \mathrm{mg} / \mathrm{kg})$ and xylazine hydrochloride $(5.1 \mathrm{mg} / \mathrm{kg})$. Additional injections of this cocktail $(1 \mathrm{ml} / \mathrm{kg})$ were repeated as required to maintain anesthesia. Rats were placed in a stereotaxic instrument (David-Kopf Instrument Co) in the flat-skull position to implant EEG electrodes. Two 22-gauge stainless steel guide cannulae were bilaterally implanted and the tip was gently lowered to $1.0-3.0 \mathrm{~mm}$ above the VLPO region $(-0.6 \mathrm{~mm}$ from bregma, \pm 1.4 from midline, $-8.8 \mathrm{~mm}$ from dura) or $1 \mathrm{~mm}$ above the hippocampus $(-4.3$ $\mathrm{mm}$ from bregma, \pm 4.0 from midline, $-3.0 \mathrm{~mm}$ from dura) to prevent damage to the target region. All electrodes and guide cannulae were secured in place with dental cement. A 27-gauge injection needle was then inserted on each side, such that the needle's tip reached into the VLPO region, and the adenoviral vector (Ad-ChR2-Kat1.3 or Ad-eGFP) or adeno-associated viral vector (AAV-ChR2-eYFP or AAV-eYFP) was injected into the animals for $10 \mathrm{~min}$ at a rate of $0.1 \mu \mathrm{l} / \mathrm{min}$. The needle was removed $10 \mathrm{~min}$ after the injection. Subsequently, a fiber optic cannula $(200 \mu \mathrm{m}$ in diameter, $0.37 \mathrm{NA})$ was inserted into each guide cannula, such that the fiber optic cannula's tip was placed $0.5 \mathrm{~mm}$ above the VLPO region, before being fixed with dental cement.

\section{Photostimulation}

After recovery from surgery, optic cables connected to a laser source ( $473 \mathrm{~nm}$; SDL-473-100MFL, Shanghai Dream Lasers Co) were connected to the optic cannulae for photostimulation. Photostimulation was applied bilaterally for 30-120 min at a frequency of $1 \mathrm{~Hz}$ ( $500 \mathrm{~ms}$ on/off) by a stimulator (Model S-88, Grass Inc.). The light intensity at the tip of the fiber optic cable was 1.3-1.7 mW (PM204, Thorlabs Co) and, usually, the light intensity at the tip of the optic cannulae was $80-85 \%$ of that at the tip of the optic cable. Sleep and behavior were recorded from 9:30 A.M. to 6:30 or 10 P.M. The first session for stimulation was done during mostly sleeping state in the light phase (12:30 to 2:30 P.M.) and another session for stimulation was done during mostly awake state in the light phase (3:30-5:30 P.M.). At least $24 \mathrm{~h}$-interval was given between the sessions. If photostimulation was to induce sleep, three 30-min stimulation sessions were performed successively (11:00-11:30 A.M., 2:002:30 P.M., and 5:00-5:30 P.M.) immediately after awakening the animal by cage tilting (one side of the cage was lifted up at an angle of $45^{\circ}$ from the floor three times) before sleep latencies were measured. Cage tilting brings rats to the same level of awakeness in a manner similar to that of the multiple sleep latency test used to measure sleepiness (McKenna et al., 2008). We employed this cage tilting method to test the effects of optogenetic stimulation on sleep promotion by measuring sleep latency. Additional photostimulation experiments were performed in both the light (12 to 2 P.M.) and dark (7-9 P.M.) phases in rats injected with AAV-ChR2-eYFP and AAV-eYFP to test the reproducibility of effects with another viral vector. Each 120-min photostimulation session in both the light and dark phases was conducted separately with at least 24$\mathrm{h}$ time interval. The hippocampus was selected as a control brain region to confirm the specific effects on VLPO astrocytes.

\section{Microinjection of drugs}

After three consecutive stable recording sessions, experimental microinjections were initiated. First, the stylet was removed from the guide cannula and then a 27-gauge injection needle was inserted on each side, such that the needle's tip extended into the VLPO region $(-0.6 \mathrm{~mm}$ from bregma, \pm 1.4 from midline, $-8.8 \mathrm{~mm}$ from dura). Subsequently, L- $\alpha$-AA, TNAP-I, or vehicle ( $3 \mu \mathrm{l}$ per side for $30 \mathrm{~min}$ at a rate of $0.1 \mu \mathrm{l} /$ $\mathrm{min}$ ) was infused through an injection needle connected to a $50 \mu \mathrm{l}$ Hamilton syringe via a length of polyethylene tubing (PE-25) and using a syringe pump (Model 22; Harvard Apparatus).

\section{Recording and sleep-wake state scoring}

Recordings were started at least $7 \mathrm{~d}$ after surgery. After the animals were acclimated to the recording setup (i.e., the chamber and connection cable) for $3 \mathrm{~d}$, the daily recording was performed from 9:30 A.M. to 6:30 or 10 P.M. EEG signals from the cortices and vibration signals from the vibration plate were amplified with $10,000 \times$ and $10 \times$ gain, respectively, and they were filtered at $0.5-100 \mathrm{~Hz}$ (Model 3500; AM Systems). The signals were digitized at a rate of $200 \mathrm{~Hz}$ (DAQ PAD6015; National Instrument Inc.) with a custom-made LabView program (National Instruments Inc.). Sleep-wake states were rated by two experienced scorers according to EEG and vibration signals, which represented motor activity. Each 10-s epoch was scored as one of three states: wake, slowwave sleep (SWS), or paradoxical sleep (PS). All procedures in the behavioral tests, including surgery, virus injection, and the scoring of sleep-wake states, were performed in a randomized double-blind manner to ensure impartiality and avoid errors arising from bias.

\section{Power spectrum analysis}

The power spectrum of each sleep-wake state was calculated in each segment after dividing recordings into $30 \mathrm{~min}$ segments, from $60 \mathrm{~min}$ before to $60 \mathrm{~min}$ after photostimulation. To evaluate sleep intensity, the $\delta$ power $(0.5-2.5 \mathrm{~Hz})$ was derived from the power spectrum of the SWS state during stimulation.

\section{Electrophysiology}

\section{Whole-cell patch-clamp recordings}

Electrophysiological measurements were performed from cultured astrocytes expressing ChR2 at a holding potential of $-80 \mathrm{mV}$ using a patchclamp amplifier (Axopatch 200B; Molecular Devices). For whole-cell recordings, patch pipettes were made from borosilicate capillary glass (1.5 mm outer diameter, $0.9 \mathrm{~mm}$ inner diameter; G-1.5; Narishige) using a pipette puller (P-97; Sutter Instrument $\mathrm{Co}$ ). The resistance of the recording pipettes filled with internal solution $(140 \mathrm{~mm}$ Cs-methanesulfonate, $10 \mathrm{~mm} \mathrm{CsCl}, 2$ mм EGTA, 2 mм Mg-ATP, and 10 mм HEPES; $\mathrm{pH}$ 7.2 with Tris-base) was 2-5 $\mathrm{M} \Omega$; the liquid junction potential was corrected. Astrocytes were viewed under phase contrast on an inverted microscope (TE-2000; Nikon). Membrane current was low-pass filtered at $1 \mathrm{kHz}$ and acquired at $4 \mathrm{kHz}$ (Digidata 1440; Molecular Devices). During recordings, $10-\mathrm{mV}$ hyperpolarizing step pulses $(30 \mathrm{~ms}$ in duration) were periodically applied to monitor the access resistance, and recordings were discontinued if access resistance changed by $>10 \%$. All 


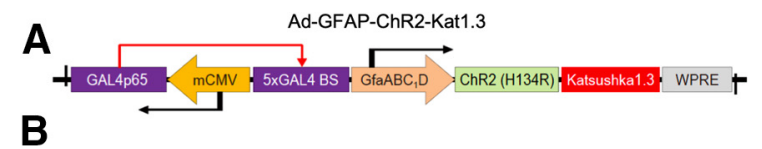

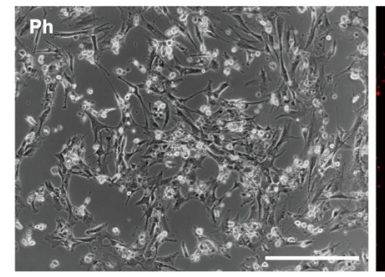

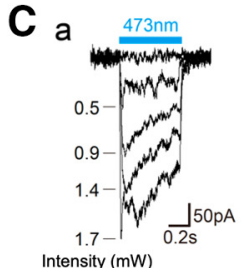

E
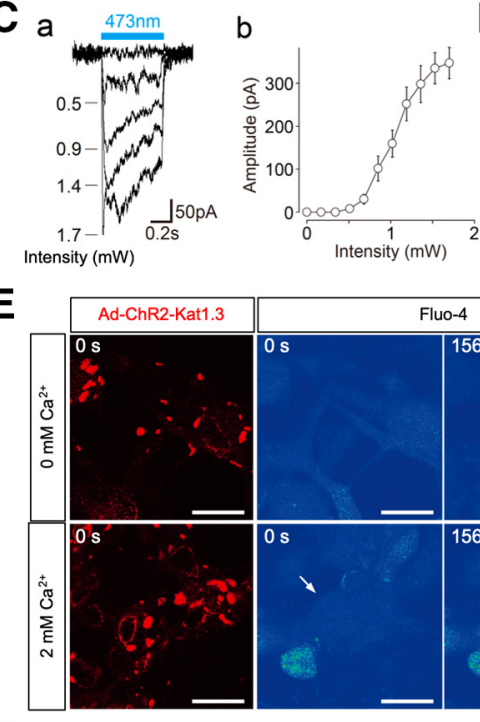

G



AAV-GFAP-ChR2-eYFP

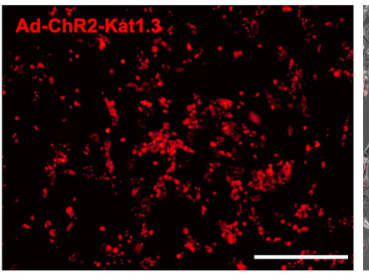

D a control $473 \mathrm{~nm}$

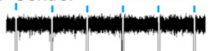



$\mathrm{Na}^{+}$-free $\left(\mathrm{NMDG}^{+}\right)$

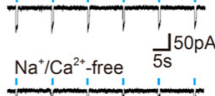

$F$
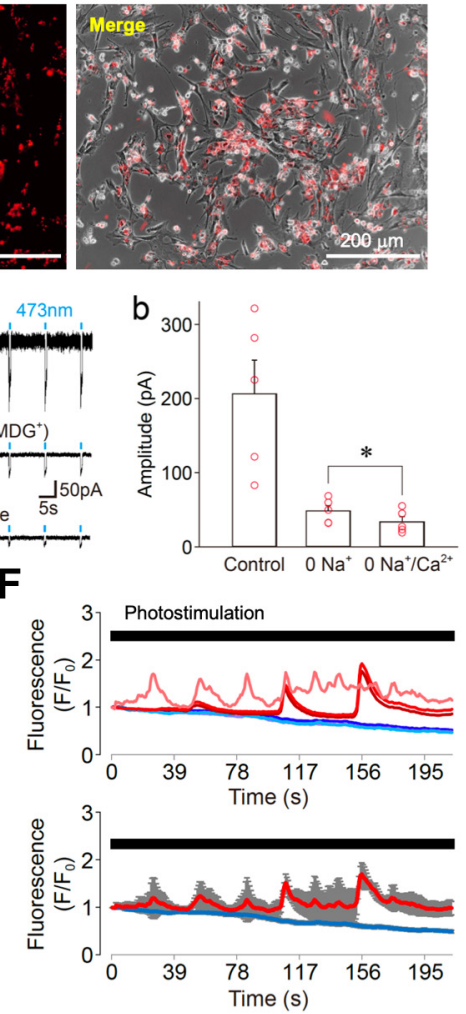

ITR hGFAP promoter hChR2(H134R) EYFP WPRE
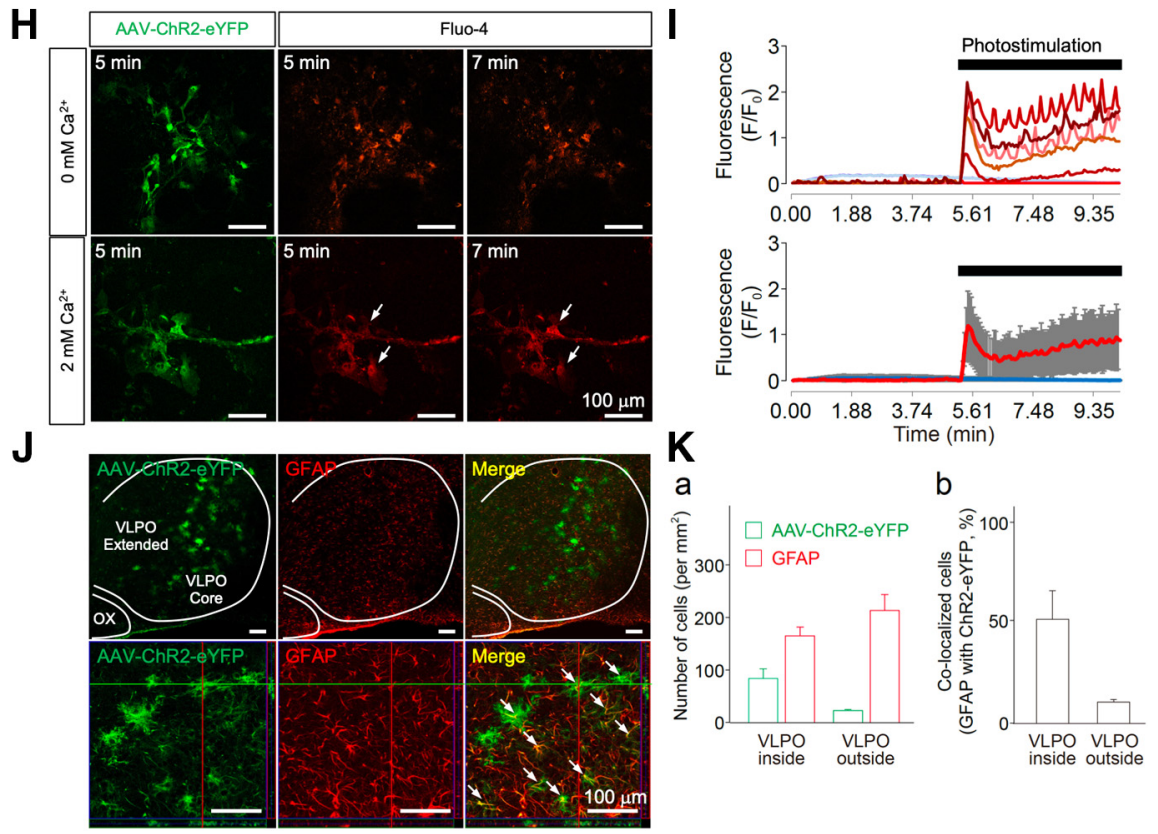

Figure 1. Functional expression of ChR2 in astrocytes. A, An illustration of the adenoviral vector containing the ChR2 construct (Ad-ChR2-Kat1.3; Ad-sGFAP-ChR2(H134R)-Katushka1.3). The expression of ChR2 is controlled by GFABC1D, a shortened version of the GFAP promoter. mCMV operating in the antisense orientation drives the expression of a chimeric transcriptional activator Gal4p56, while the specificity of expression in both directions is determined by GfaABC1D. B. Phase contrast (Ph, left), fluorescence (middle), and superimposed (right) images of cultured astrocytes infected with Ad-ChR2-Kat1.3. C, Typical traces of membrane currents induced by different photostimulation ( $473 \mathrm{~nm}$ ) intensities in cultured astrocytes (a). Photostimulation intensity-current relationship in cultured astrocytes $(\boldsymbol{b})$. All points and error bars represent the mean and SEM from four experiments. $\boldsymbol{D}$, Typical traces of photostimulation (473 nm)-induced membrane currents in a standard external solution (upper), a $\mathrm{Na}^{+}$-free (replaced with equimolar $\left.\mathrm{NMDG}^{+}\right)$solution $\left(0 \mathrm{Na}^{+}\right.$; middle), and a solution that was both $\mathrm{Na}^{+}$-free and $\mathrm{Ca}^{2+}$-free $\left(0 \mathrm{Na}^{+} / \mathrm{Ca}^{2+}\right.$; lower; $\left.\boldsymbol{a}\right)$. Changes in photostimulation-induced membrane currents for each condition $(\boldsymbol{b})$. All columns and error bars represent the mean and SEM from five experiments. Note that the photostimulation-induced membrane currents in the $\mathrm{Na}^{+}$-free solution were significantly reduced by omission of extracellular $\mathrm{Ca}^{2+} ; * p<0.05 ;$ paired $t$ test; $t_{(4)}=4.21, p=0.0136$. $\boldsymbol{E}$, Typical fluorescence images of ChR2-expressing astrocytes displaying the $\mathrm{Ca}^{2+}$ response on photostimulation. Cultured astrocytes were infected with Ad-ChR2-Kat1.3 and loaded with Fluo- 
experiments were performed at room temperature $\left(22-24^{\circ} \mathrm{C}\right)$. The external solution ( $150 \mathrm{~mm} \mathrm{NaCl}, 3 \mathrm{~mm} \mathrm{KCl}, 2 \mathrm{~mm} \mathrm{CaCl}$, $1 \mathrm{~mm} \mathrm{MgCl}_{2}, 10 \mathrm{~mm}$ glucose, and $10 \mathrm{~mm}$ HEPES; pH 7.4 with Tris-base) was applied using the "Y-tube system" for rapid solution exchange (Murase et al., 1989). In the $\mathrm{Na}^{+}$-free external solution, $\mathrm{NaCl}$ was replaced with equimolar $\mathrm{N}$ methyl-D-glucamine-Cl. A fiber optic cannula (200- $\mu \mathrm{m}$ inner diameter, $0.37 \mathrm{NA}$ ) connected to a laser source [blue laser $(473 \mathrm{~nm})$; SDL-473100MFL; Shanghai Dream Lasers Technology Co] was placed on the vicinity of astrocytes (within $500 \mu \mathrm{m}$ ) using a micromanipulator. Photostimulation (500 $\mathrm{ms}$ in duration) was triggered at a frequency of $0.1 \mathrm{~Hz}$ using a stimulator (SEN-7203; Nihon Kohden). The maximal light intensity at the tip of the fiber optic cannula was $1.7 \mathrm{~mW}$ (PM204; Thorlabs Co).

\section{Statistics}

The parametric statistical test methods used in this study were twotailed paired or unpaired Student's $t$ tests and one-way ANOVA. Each test method was chosen based on the properties of the data under the assumption that they had a normal distribution. All statistical test methods are defined in the relevant figure legends. Changes in ATP or adenosine concentration and fluorescence intensity were tested using a one-way ANOVA or unpaired Student's $t$ test. In behavioral experiments, changes in the duration of sleep stage, sleep latency and $\delta$ EEG power were tested using an unpaired Student's $t$ test in the presence or absence of photostimulation. Power calculations were performed to estimate the proper sample size using the G*Power 3.1 program (Faul et al., 2009). The proper sample sizes were calculated for a power of 0.8 in all tests, and the sample sizes of data shown in this study were revealed to be satisfactory in most experimental results. Numerical values are provided as the mean \pm SEM, except where indicated. Differences were considered statistically significant at $p<0.05$.

4AM. Note that photostimulation induced a profound increase in intracellular $\mathrm{Ca}^{2+}$ concentration (arrows) in the presence of $2 \mathrm{~mm}$ extracellular $\mathrm{Ca}^{2+} . \boldsymbol{F}$, Time courses of individual (upper) and mean (lower) Fluo-4AM fluorescence intensity changes obtained from three astrocytes on photostimulation in the absence or presence of extracellular $\mathrm{Ca}^{2+}$ (blue lines, 0 $\mathrm{mm}$; red lines, $2 \mathrm{~mm}$ ). Photostimulation $(1.7 \mathrm{~mW}, 1 \mathrm{~Hz}$, horizontal bar) was applied to Fluo4AM-loaded astrocytes. Fluorescence intensities were measured using Zeiss ZEN Microscope software and are expressed as the ratio of $\left(F-F_{0}\right) / F_{0}$, where $F_{0}$ is the base level fluorescence intensity in cell bodies before any treatment. All measurements were corrected for background fluorescence. Increases in the fluorescence ratio that were $>0.1$ were considered to be significant changes; basal level fluorescence values exhibited a peak $\left(F-F_{0}\right) / F_{0}$ ratio of $0.01 \pm 0.01$ on average. The results are representative of three experiments. $\mathbf{G}$, An illustration of the adeno-associated viral vector containing the ChR2 construct (AAV-ChR2-eYFP; AAV-GFAP-hChR2(H134R)-eYFP). The expression of ChR2 is controlled by GfaABC1D, a shortened version of the GFAP promoter. $\boldsymbol{H}$, Typical fluorescence images of AAV-ChR2-eYFPexpressing astrocytes displaying a $\mathrm{Ca}^{2+}$ response on photostimulation. Cultured astrocytes were infected with AAV-ChR2-eYFP and loaded with rhodamine 2. Note that photostimulation induced a profound increase in intracellular $\mathrm{Ca}^{2+}$ concentration (arrows) in the presence of $2 \mathrm{~mm}$ extracellular $\mathrm{Ca}^{2+} . I$, Changes in individual (upper) and mean (lower) rhodamine 2 fluorescence intensities obtained from six astrocytes on photostimulation in the absence or presence of extracellular $\mathrm{Ca}^{2+}$ (blue lines, $0 \mathrm{~mm}$; red lines, $2 \mathrm{~mm}$ ). Photostimulation (1.7 $\mathrm{mW}, 1 \mathrm{~Hz}$, horizontal bar) was applied to rhodamine 2-loaded astrocytes. The results are representative of three experiments. J, Fluorescence images displaying AAV-ChR2-eYFP expression in the VLPO area. The VLPO regions in the brain slices were subjected to immunofluorescence analysis to identify AAV-ChR2-eYFP (green) expression in astrocytes (GFAP, red), which was confirmed by reconstructed Z-section images (bottom panels, highpower images). Arrows indicate the co-localization of AAV-ChR2-eYFP and GFAP. ChR2 expression (green) was not co-localized with either Iba-1 or NeuN. Dotted lines indicate the VLPO (core and extended region) and optic chiasm (OX) areas. The results are representative of six experiments. $\boldsymbol{K}$, Quantification of AAV-ChR2-eYFP-positive and GFAP-positive cells around the VLPO region. Shown are the number of cells $(\boldsymbol{a})$ and their co-localization $(\boldsymbol{b})$. Each column and error bar represents the mean and SD from three experiments.

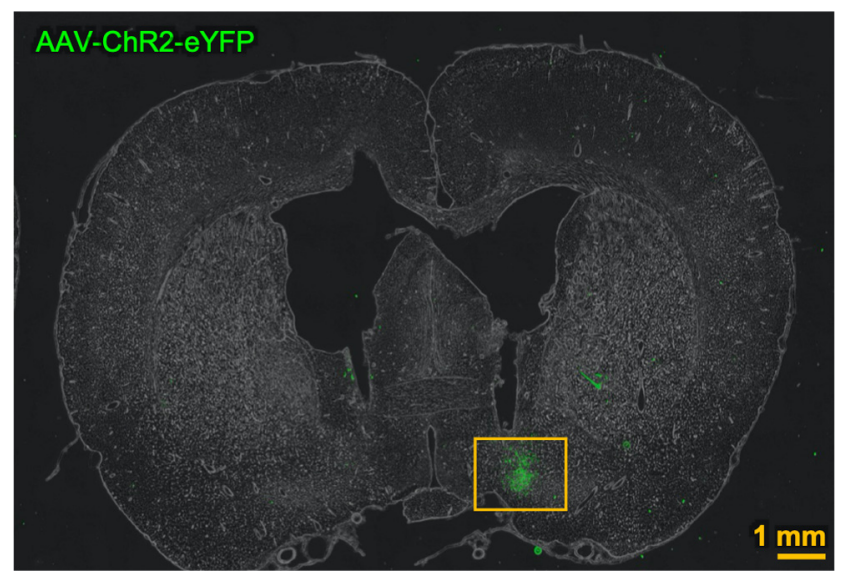

Figure 2. A representative low-magnification image showing the localization of AAVChR2-eYFP in VLPO region.

\section{Results}

Optogenetic stimulation of VLPO astrocytes promotes sleep To examine whether astrocytes within the VLPO region can promote sleep, we used optogenetic tools to selectively modulate astrocytic activity without directly affecting neighboring neurons (Figueiredo et al., 2011). First, ChR2, a light-sensitive cation channel, was selectively expressed in cultured astrocytes via viral vectors (Ad-ChR2-Kat1.3 or AAV-ChR2-eYFP). Photostimulation (at a wavelength of $473 \mathrm{~nm}$ ) through an optic fiber induced inwardly directed membrane currents in these cultured astrocytes, which indicated their functional expression of ChR2 (Fig. 1). In ChR2expressing astrocytes, photostimulation induced inwardly directed membrane currents in an intensity-dependent manner, and the maximal amplitudes were elicited by photostimulation at 1.4-1.7 $\mathrm{mW}$ (Fig. 1C). Therefore, an intensity of $1.7 \mathrm{~mW}$ was used to stimulate ChR2-expressing astrocytes in all subsequent experiments. The photostimulation-induced membrane currents were greatly reduced in the $\mathrm{Na}^{+}$-free (equimolar substitution by $\mathrm{NMDG}^{+}$) external solution, and they were further decreased by depleting extracellular $\mathrm{Ca}^{2+}$ (Fig. 1D), which suggests that ChR2 might also be permeable to $\mathrm{Ca}^{2+}$ (Timofeev and Chauvette, 2017). It has been reported that the blue light-gated channelrhodopsin pore is quite large (6.2 A) compared with the red light-gated Chrimson (2.6 A; Oda et al., 2018). Moreover, photostimulation elicited an instantaneous increase in intracellular $\mathrm{Ca}^{2+}$ concentration and sustained $\mathrm{Ca}^{2+}$ oscillations (Fig. 1E-I).

Next, we injected AAV-ChR2-eYFP (Figs. 1J,K, 2, 3) or AdChR2-Kat1.3 (Figs. $4 A-C, 5,6)$ into the VLPO region of rats using a stereotaxic system. Seven days after virus injection, photostimulation was delivered via an optic fiber to ChR2-expressing astrocytes within the VLPO region. Electroencephalogram (EEG) recordings were then taken from these animals to examine their sleep patterns (Figs. 7, 8). The spatial expression profile of ChR2 within the VLPO region as well as the astrocytic expression of ChR2 was verified after the completion of each experiment [AAV-ChR2-eYFP (Fig. 1J,K); Ad-ChR2-Kat1.3 (Fig. 4A$C)$ ]. When the animals were mostly sleeping in the light phase [Ad-ChR2-Kat1.3: wake duration $=18.5 \pm 2.8 \%$, SWS duration $=$ $58.9 \pm 2.1 \%$, and PS duration $=24.7 \pm 11.1 \%$ (Fig. $4 E$ ); AAVChR2-eYFP: wake duration $=43.4 \pm 21.1 \%$, SWS duration $=$ $40.5 \pm 18.8 \%$, and PS duration $=15.9 \pm 3.4 \%$ (Fig. $8 C$ ) , photostimulation did not significantly change sleep and/or wake durations (Figs. 4E, 8C) and sleep latency (Fig. 7Bd1). In contrast, when animals were mostly awake during later period in the light 
A
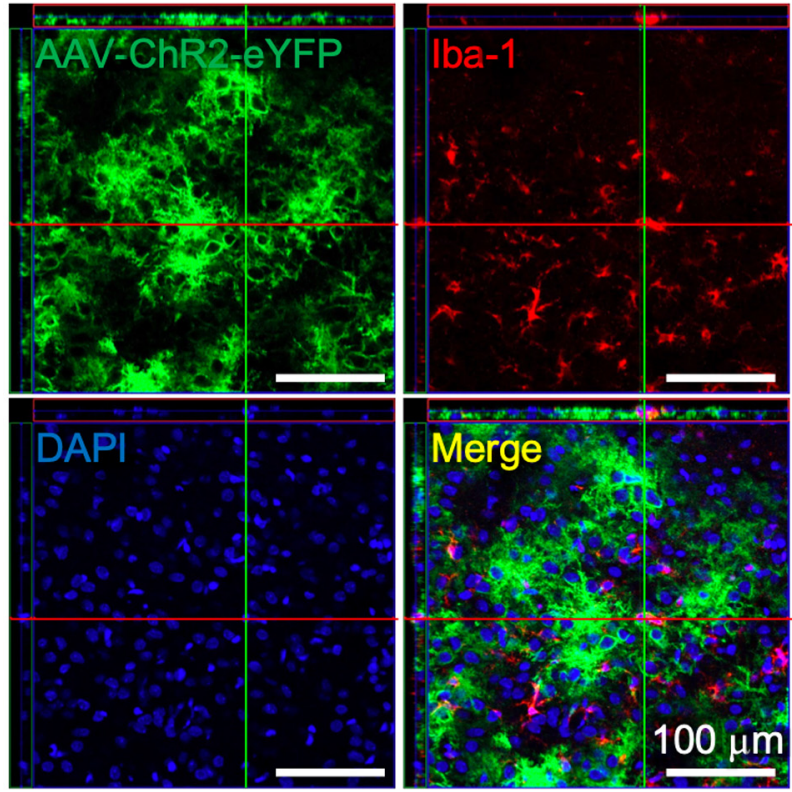

C
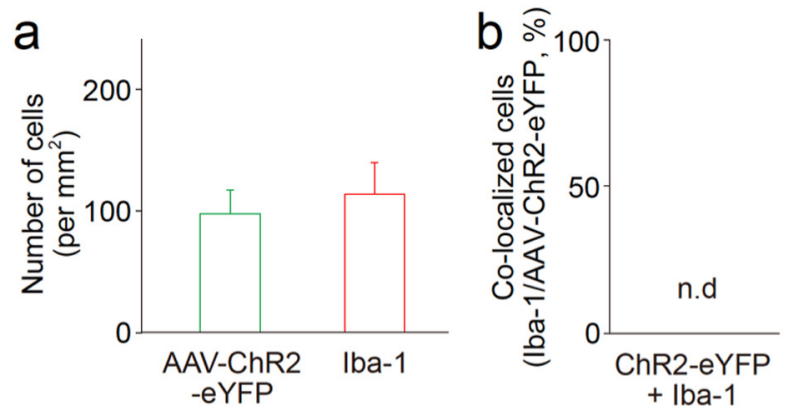

B
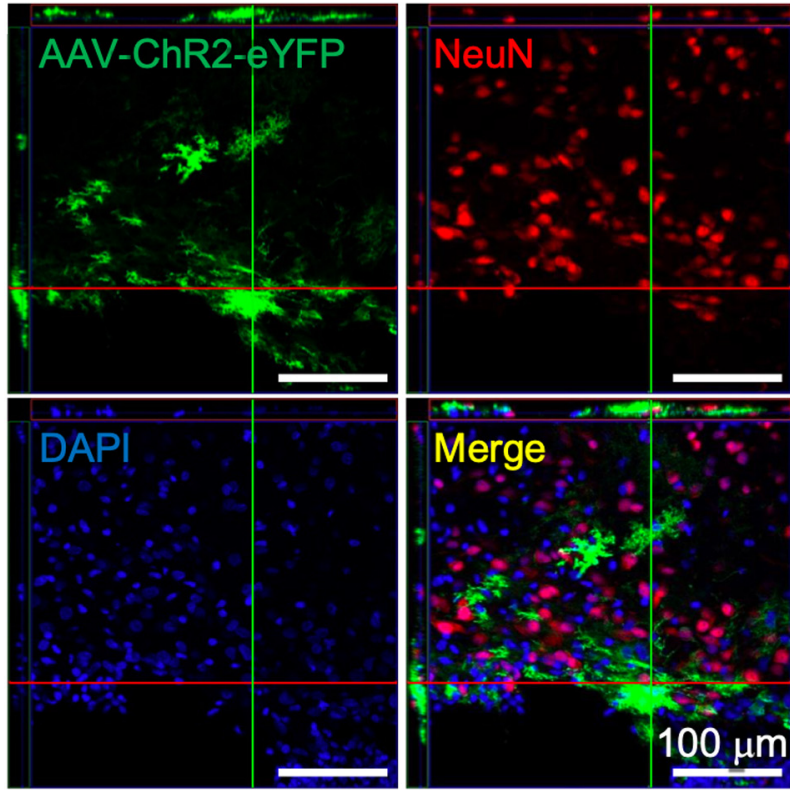

D a

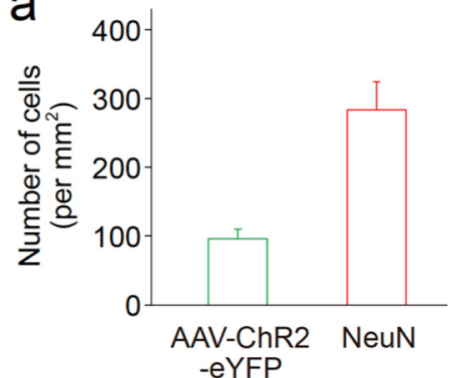

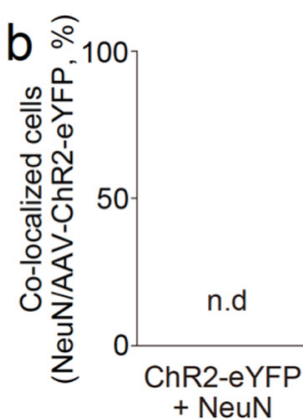

Figure 3. Expression of ChR2 in astrocytes in the VLPO area following AAV-mediated gene transfer. $\boldsymbol{A}, \boldsymbol{B}$, The VLPO regions of rat brains infected with AAV-ChR2-eYFP were subjected to immunofluorescence analysis to evaluate ChR2 (green) expression in microglia ( $\boldsymbol{A}$, lba-1, red) and neurons ( $\boldsymbol{B}$, NeuN, red). These results were confirmed by reconstructed Z-section images. Nuclei were stained with DAPI (blue). Images are representative of three independent experiments. C, D, Quantification of Iba-1, NeuN, or AAV-ChR2-eYFP-positive cells around the VLPO region. Shown are the number of cells $(\boldsymbol{a})$ and their co-localization $(\boldsymbol{b})$. Each column and error bar represents the mean and SD from three experiments; n.d., not detected. Note that neither Iba-1 nor NeuN was co-localized with AAV-ChR2-eYFP.

phase (Ad-ChR2-Kat1.3: wake duration $=59.1 \pm 13.7 \%$, SWS duration $=35.7 \pm 6.8 \%$, and PS duration $5.1 \pm 6.9 \%$; Fig. $4 F$ ) or dark phase (AAV-ChR2-eYFP: wake duration $=82.9 \pm 6.7 \%$; SWS duration $=12.5 \pm 6.6 \%$; and PS duration $=4.5 \pm 3.1 \%$; Fig. $8 D$ ), photostimulation significantly increased SWS and PS durations ( $p<0.05$; Figs. $4 F, 8 D$ ) and decreased sleep latency (Fig. $7 B d 1)$. Such effects were not observed in rats injected with a control viral vector (Ad-eGFP or AAV-eYFP; Figs. 7Ba2-d2, 8G-I). Photostimulation did not increase $\delta$ power during SWS, although sleep duration was increased (Fig. $4 E, F b, 7 C, 8 E, F$ ). In addition, photostimulation of the hippocampus, which is not related to sleep control, did not promote sleep, consistent with the specific sleep-promoting effect of VLPO photostimulation (Fig. 8J).

\section{An increase in the activity of VLPO astrocytes and ATP} levels during sleep states

Given that our results thus far showed that the optogenetic activation of VLPO astrocytes was likely to promote sleep, we next determined whether the activity of VLPO astrocytes changed during natural sleep. A previous study showed that levels of cFos expression within the VLPO area are proportional to sleep duration (Sherin et al., 1996); therefore, we examined the expression of c-Fos protein within the VLPO region in light and dark phases. The total number of c-Fos-positive cells was significantly higher in the light phase relative to the dark phase $(90.0 \pm 22.3$ and $33.0 \pm 22.8$ cells in the light and dark phases, respectively; $n=4$; $p<0.05$; Fig. 9E). The number of cells co-localized with cFos and GFAP was also significantly higher in the light phase than in the dark phase $(27.5 \pm 9.6$ and $5.8 \pm 1.7$ in light and dark phases, respectively; $n=4 ; p<0.05$; Fig. $9 E)$. These results suggest that a causal relationship exists between the increase in the activity of VLPO astrocytes and natural sleep behavior. We also examined whether the increase in astrocyte activity affects neuronal c-Fos expression within the VLPO region. In rats for which AAV-ChR2-eYFP was injected into the VLPO region, photostimulation of VLPO astrocytes significantly increased the number of cells co-localized with c-Fos and GAD67, a GABAergic neuronal marker, within the VLPO region ( $48 \pm 8$ and $68 \pm 12$ cells in the unstimulated and photostimulation groups, respectively; $n=3$; $p<0.01$; Fig. 9F). Moreover, photostimulation significantly increased the number of cells co-localized with c-Fos and GFAP (5.1 \pm 4.0 and $27.1 \pm 7.1$ cells in the unstimulated and photostimulation groups, respectively; $n=3$; $p<0.05$; Fig. $9 G$ ). 
EEG
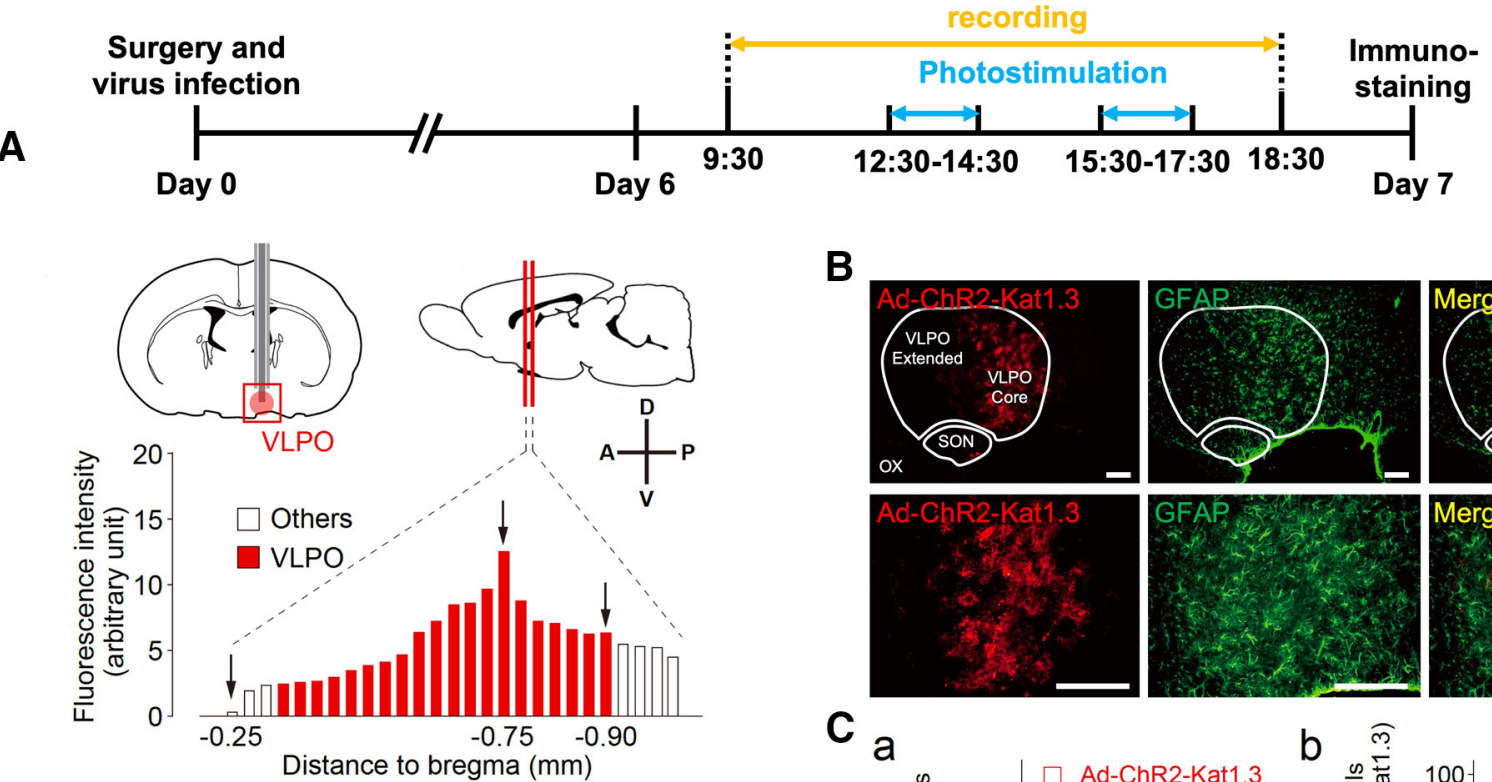

B
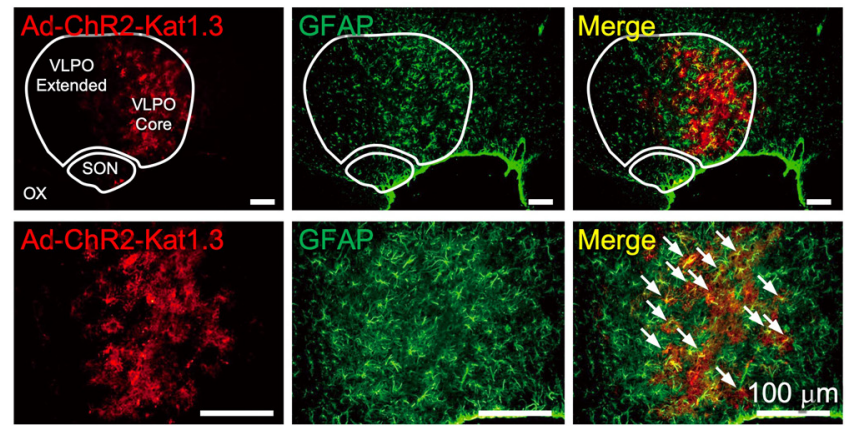

C
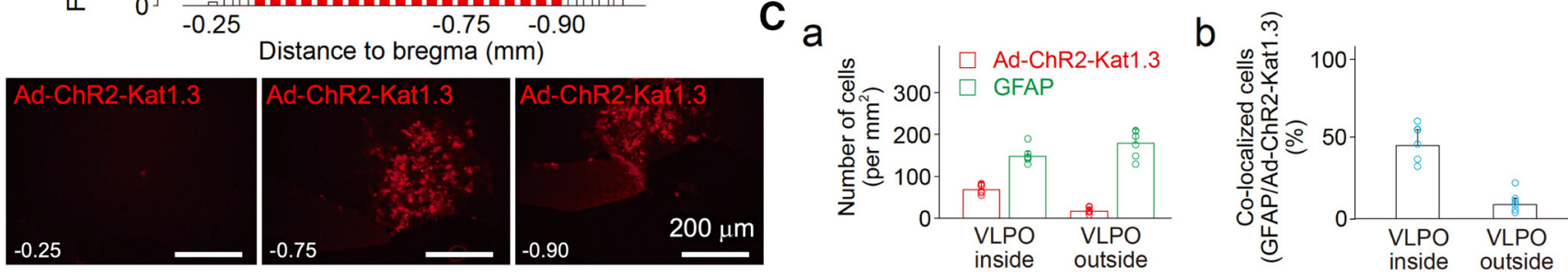

D

15:00

473nm (15:30-17:30)

17:30

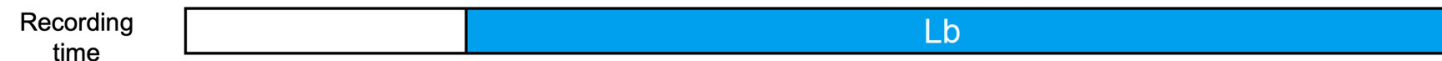

FC EEG

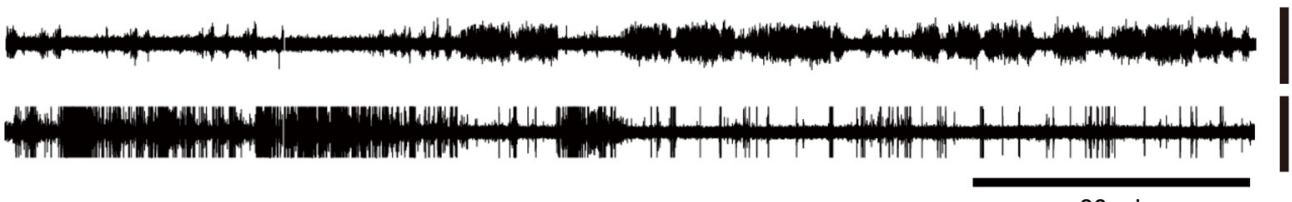

$0.1 \mathrm{mV}$

Vibration

$30 \mathrm{~min}$

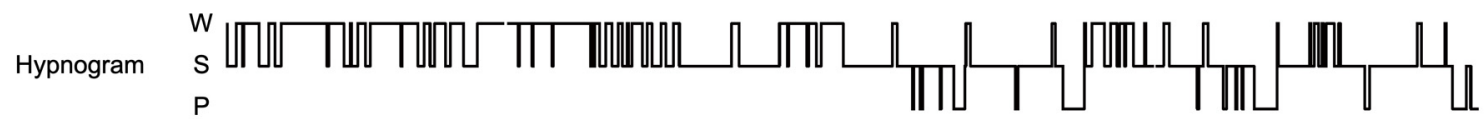

E

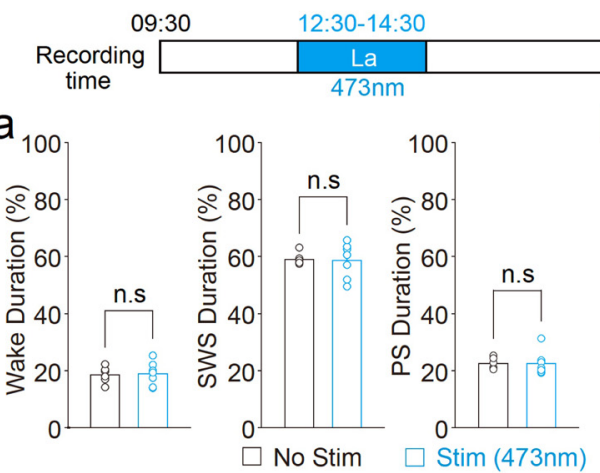

$18: 30$

b

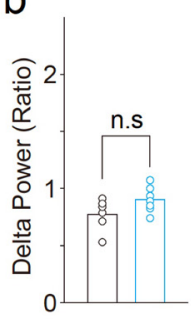

$\mathbf{F}$

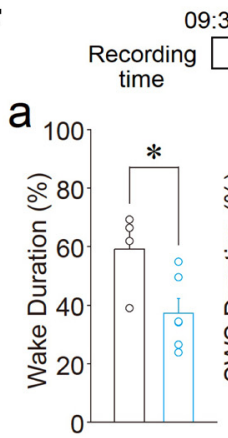

$9: 30$
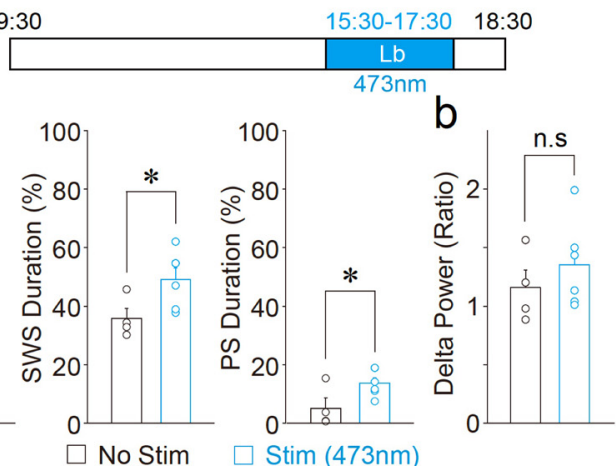

Figure 4. Optogenetic stimulation of the VLPO astrocytes promotes sleep when rats are in a waking state. $\boldsymbol{A}$, Timeline of experiments (top). Visual representation of the cannula placement site used to stimulate VLPO astrocytes and the quantification of the ChR2-Kat1.3 expression profile in the VLPO area. Ad-ChR2-Kat1.3 was delivered to the VLPO area using a guide cannula. The expression of ChR2 fused to Katushka1.3 was confirmed by fluorescence (red) detected in serial coronal sections of the brain. The VLP0 area corresponds to the position $0.3-0.9 \mathrm{~mm}$ posterior to bregma (red columns). The graph shows the quantification of ChR2 expression in the serial sections. Representative fluorescence images are also shown in the lower panel. Expression of Ad-ChR2-Kat1.3 was confirmed in 15 animals, among which four animals were subjected to serial brain sectioning and fluorescence analysis. $\boldsymbol{B}$, Fluorescence images of the VLPO region. Note that Ad-ChR2-Kat1.3 (red) is co-localized with GFAP (green). Dotted lines indicate the areas of the VLPO region (core and extended region), supraoptic nucleus (SON), and optic chiasm (OX). The results are representative of six experiments. Arrows in the magnified images (lower row) indicate co-localization of Ad-ChR2-Kat1.3 and GFAP. C, Quantification of cells positive for AdChR2-Kat1.3 and GFAP around the VLP0 region. Shown are the number of cells $(\boldsymbol{a})$ and their co-localization $(\boldsymbol{b})$. Each column and error bar represents the mean and SD from six experiments. $D$, Representative traces of a frontal cortex EEG (FC EEG), motor activity-related vibration, and a hypnogram before and during (Lb) photostimulation. W, wake; S, SWS; and P, PS. E, Effects of photostimulation during the 12:30 P.M. to 2:30 P.M. (La) period. The duration of sleep-wake states $(\boldsymbol{a})$ in the stimulation group (Stim, $n=7$ ) was similar to that of the control group (No Stim, 


\section{Identification of sleep-regulating gliotransmitters within the VLPO region}

Our results showed that c-Fos expression in VLPO astrocytes was related to sleep-wake states and that photostimulation of ChR2-expressing astrocytes increased c-Fos expression in VLPO neurons, which suggests that a possible interaction exists between astrocytes and neurons within the VLPO region. Since a neuron-astrocyte interaction could be mediated by gliotransmitters (Araque et al., 2014), we attempted to determine which gliotransmitters were released from VLPO astrocytes. Seven days after an injection of Ad-ChR2Kat1.3 into the VLPO region of living rats, photostimulation was applied to VLPO astrocytes; subsequently, ECFs in the VLPO area were obtained using a microdialysis technique (Figs. 10A, 11). Bioluminescence analysis of the microdialysate collected during $120 \mathrm{~min}$ of photostimulation revealed that the ATP concentration in the ECF of the ChR2-expressing test group was significantly higher than in that of the control group, which did not express ChR2 (54.0 \pm 8.5 and $6.1 \pm 0.6 \mathrm{nM}$ in the test and control groups, respectively; $n=3 ; p<0.01$; Fig. 10B). This photostimulation-induced increase in ATP concentration was greatly attenuated by pretreatment of the VLPO region with L- $\alpha$-AA ( $3 \mu \mathrm{l}, 10 \mathrm{~nm}$ ), a metabolic inhibitor of astrocytes (Jiang et al., 2016; Nam et al., 2016; Fig. 10C), suggesting that astrocytes were the major cellular source of ATP in our in vivo experiments. In contrast to ATP, the concentrations of other known gliotransmitters, i.e., glutamate, D-serine, and glycine, were not increased in microdialysates obtained from the VLPO area during photostimulation (Fig. 10D). ATP has been previously reported to induce IL- $1 \beta$ and TNF- $\alpha$ release from glia via P2X receptors (Hide et al., 2000; Suzuki et al., 2004; Bianco et al., 2005). Thus, in addition to adenosine, these cytokines may have also increased sleep. However, an increase in the TNF- $\alpha$ and IL- $1 \beta$ levels in the dialysate samples was not detected by ELISA either in VLPO or hippocampus (Fig. 12). Although it is certainly possible that the cytokines may be released and exert sleep-promoting effects, the difference in the cytokine levels may be below the detection limit in our hand.

We also examined whether ATP concentration changed during natural wake-sleep states using a microdialysis technique coupled with EEG recording. We found that ATP concentration was significantly higher in microdialysates obtained from the VLPO region during a light phase relative to those obtained during a dark phase $(55.8 \pm 3.5$ and $11.2 \pm 3.1 \mathrm{~nm}$ from light and dark phases, respectively; $n=4 ; p<0.05$; Fig. $10 E, F)$. However, the increase in ATP concentration in microdialysates from a light phase was greatly reduced to $23.2 \pm 3.0 \mathrm{~nm}$ by the pretreatment of the VLPO region with L- $\alpha$-AA ( $3 \mu$ l, 10 nм; $n=4, p<0.05$; Fig. $10 E)$, suggesting that ATP might be released from astrocytes during natural sleep states. To confirm whether the release of ATP from VLPO astrocytes was related to sleep-

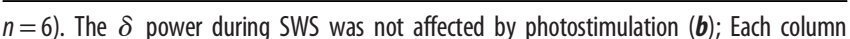
and error bar represents the mean and SD from 6-7 experiments; n.S., not significant; unpaired $t$ test. $\boldsymbol{F}$, Effects of photostimulation during the 3:30 to 5:30 P.M. (Lb) period. The duration of sleep $(\boldsymbol{a})$ in the stimulation group (Stim, $n=6$ ) was increased relative to that in the control group (No Stim, $n=4$ ). The $\delta$ power during SWS was not affected by photostimulation $(\boldsymbol{b})$; Each column and error bar represents the mean and SD from 4-6 experiments; ${ }^{*} p<0.05$; n.S., not significant; unpaired $t$ test; Wake, $t_{(8)}=2.62, p=0.0305$; SWS, $t_{(8)}=$ $2.37, p=0.0446 ; \mathrm{PS}, t_{(8)}=2.37, p=0.0453$.

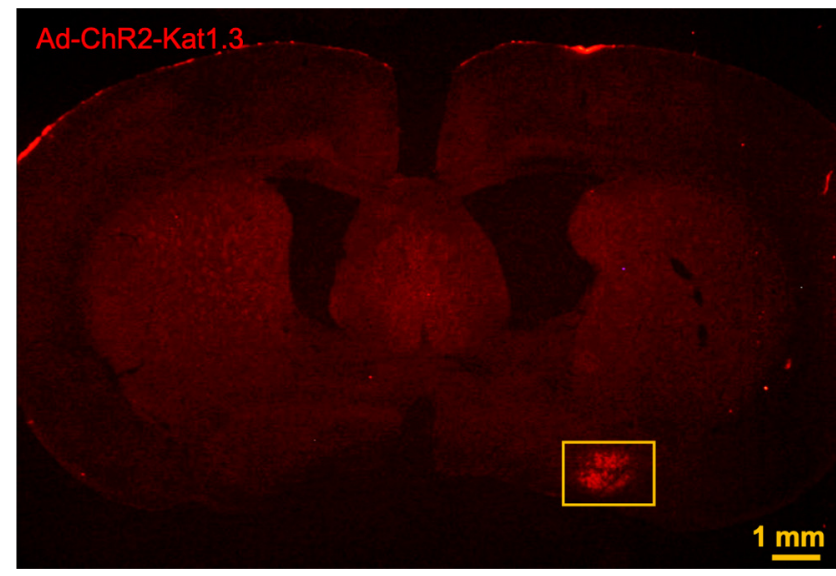

Figure 5. A representative low-magnification image showing the localization of Ad-ChR2Kat1.3 in VLP0 region.

wake states, we examined the effects of L- $\alpha$-AA on sleepwake states in living animals. We found that the infusion of $\mathrm{L}-\alpha$-AA $(3 \mu \mathrm{l}, 10 \mathrm{nM})$ into the VLPO region increased the animals' wake duration $(26.1 \pm 2.1 \%$ and $81.0 \pm 9.6 \%$ in the control and L- $\alpha$-AA groups, respectively; $n=4 ; p<0.05$ ) and decreased their total sleep duration (Fig. 10G-I).

ATP can be released from astrocytes via either P2X7 receptors or hemichannels (Bennett et al., 2003; Suadicani et al., 2006; Gandelman et al., 2010; Xing et al., 2019); therefore, we examined the effects of Brilliant Blue G (BBG), a P2X7 receptor antagonist, and carbenoxolone (CBX), a hemichannel blocker (Fujii et al., 2017; Xiong et al., 2018), on the photostimulation-induced ATP release in cultured astrocytes. We found that the photostimulation-induced ATP release was significantly attenuated by either $100 \mathrm{~nm}$ BBG or $3 \mu \mathrm{m} \mathrm{CBX} \mathrm{(Fig.} \mathrm{13),} \mathrm{suggesting} \mathrm{that} \mathrm{ATP} \mathrm{is}$ released from astrocytes through hemichannels and/or P2X7 receptors.

\section{Conversion of ATP to adenosine via TNAP in the VLPO}

ATP released from astrocytes may be hydrolyzed into adenosine by ATP-degrading enzymes, including ectonucleoside triphosphate diphosphohydrolase (NTPDase) and/or TNAP. Since TNAP is abundantly expressed within the brain and is responsible for the hydrolysis of extracellular ATP to adenosine in several brain regions (Dahl et al., 2009), we examined the role of TNAP in the VLPO. With the aim of determining the roles of both TNAP activity and the hydrolysis of ATP into adenosine in sleep-wake states, we examined whether adenosine concentration changes during natural sleep-wake states. We found that the adenosine concentration in microdialysates obtained from the VLPO region during a light phase was significantly higher than in those obtained during a dark phase $(364.0 \pm 53.2$ and $204.0 \pm 96.3 \mathrm{~nm}$ in the light and dark phases, respectively; $n=5 ; p<0.05$; Fig. $14 A, B)$. However, the increase in adenosine concentration in microdialysates during the light phase was greatly reduced to $200.2 \pm 25.4 \mathrm{~nm}$ by pretreatment of the VLPO region with TNAP-I $(3 \mu \mathrm{l}, 10 \mathrm{mg} / \mathrm{ml} ; n=5, p<0.05$; Fig. $14 A)$, which suggests that TNAP is responsible for the adenosine concentration increase within the VLPO region during natural sleep states. We also examined the effect of TNAP-I on sleep-wake states in living animals to confirm whether the ATP hydrolysis by TNAP is critical to these states. We found that the infusion of TNAP-I $(3 \mu \mathrm{l}, 10 \mathrm{mg} / \mathrm{ml})$ into the VLPO region 
A
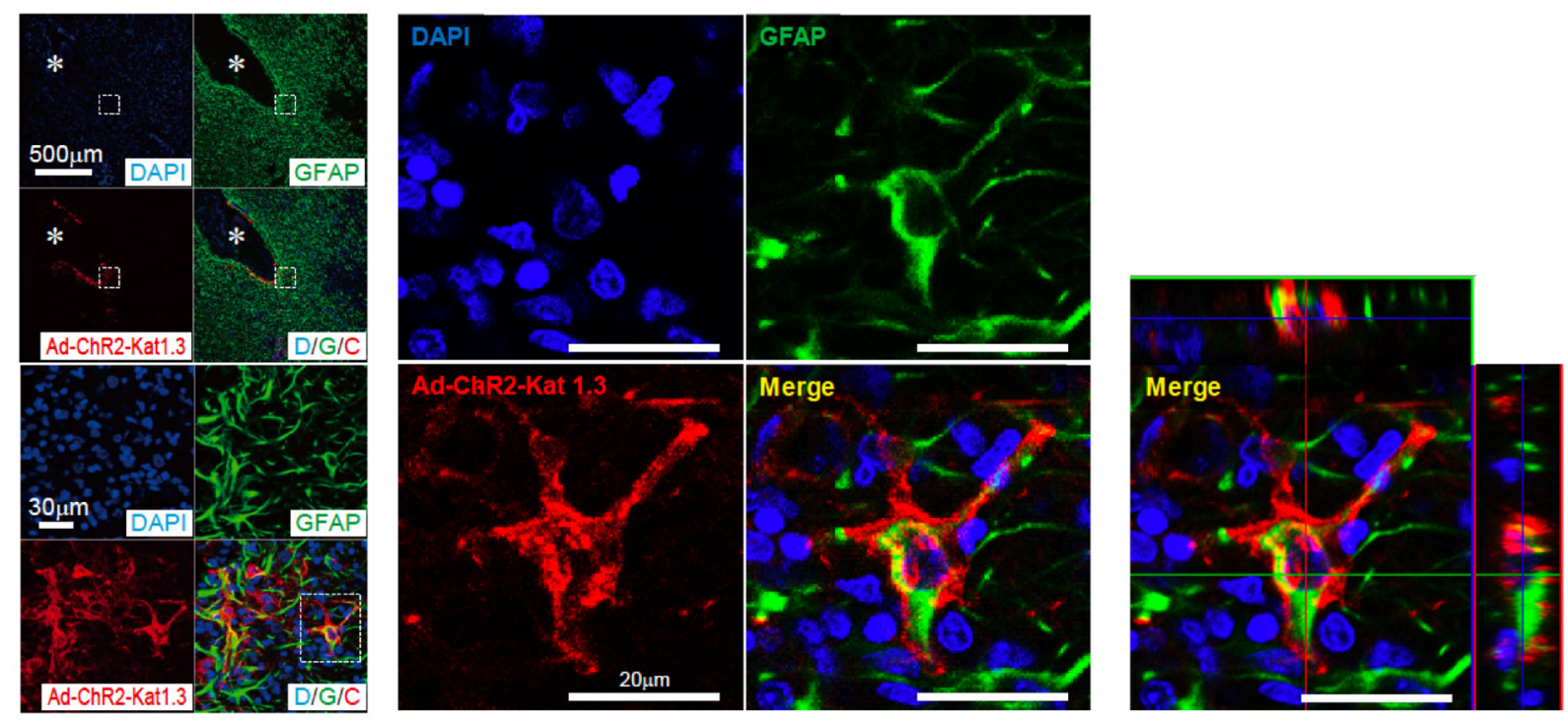

B

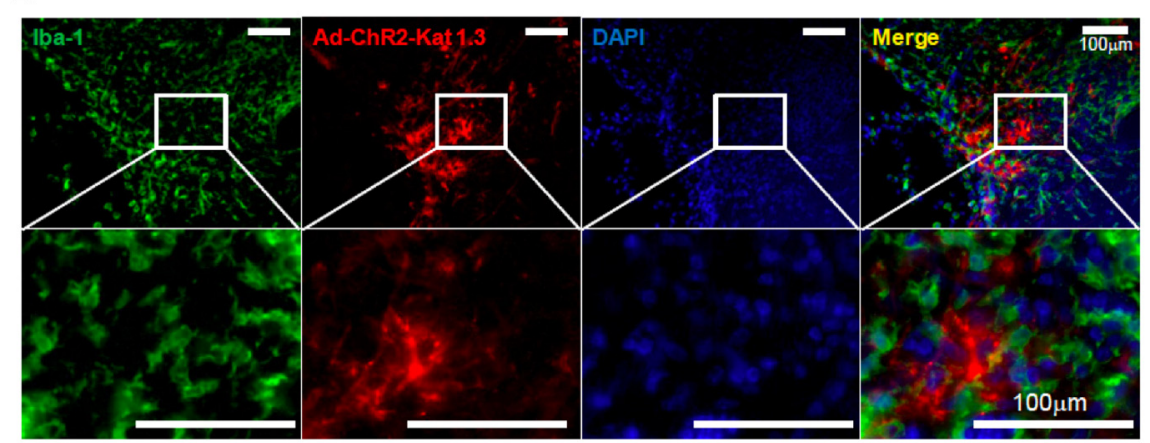

D
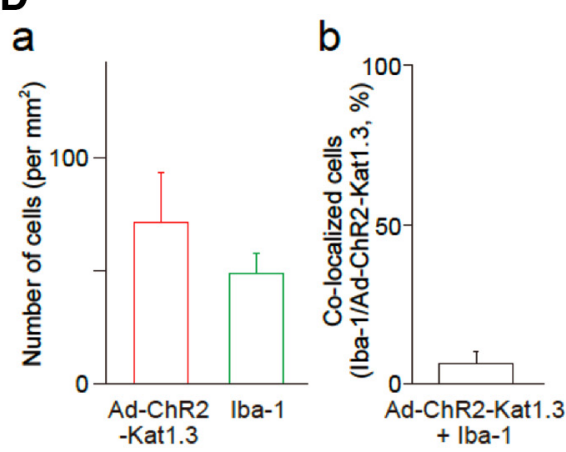

C

$E$
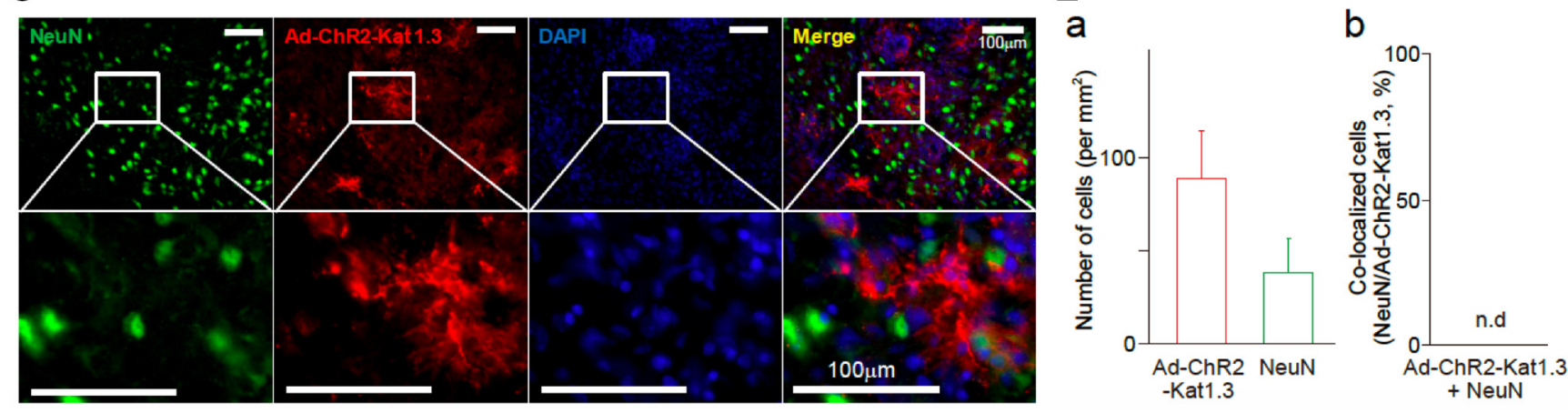

Figure 6. Expression of ChR2 in astrocytes in the VLPO area following Ad-mediated gene transfer. A, Astrocytic expression of Ad-ChR2 (Ad-ChR2-Kat1.3) was confirmed by co-localization of GFAP (green) staining and ChR2-Kat1.3 (red) expression in the VLP0 area. Asterisks indicate the virus injection sites. ChR2-Kat1.3 (red) was found in the membrane of GFAP-positive astrocytes (green), which was confirmed by reconstructed Z-section images. Nuclei were stained with DAPI (blue). The results are representative of five experiments. $\boldsymbol{B}, \boldsymbol{C}$, Brain sections containing the VLPO region were subjected to immunofluorescence analysis to assess the expression of ChR2 in microglia or neurons. ChR2 expression (red) was not co-localized with either Iba-1 (B, green, a microglial marker) or NeuN (C, green, a neuronal marker). Nuclei were stained with DAPI (blue). The results are representative of five experiments. $\boldsymbol{D}, \boldsymbol{E}$, Quantification of cells positive for Iba1, NeuN, or ChR2-Kat1.3 around the VLPO region. Shown are the number of cells $(\boldsymbol{a})$ and their co-localization $(\boldsymbol{b})$. Each column and error bar represents the mean and SD from five experiments; n.d., not detected.

increased the animals' wake duration $(16.0 \pm 3.6 \%$ and $42.7 \pm 10.8 \%$ in the control and TNAP-I groups, respectively; $n=5 ; p<0.05$ ) and decreased total sleep duration (Fig. $14 C-E)$. Thus, both the hydrolysis of ATP released from VLPO astrocytes by TNAP activity and the resultant adenosine apparently play critical roles in the sleep-promoting effects on VLPO neurons.

\section{Discussion}

In the present study, we demonstrate the process of astrocytemediated sleep promotion in the VLPO region. Optogenetic gain-of-function study shows that selective stimulation of VLPO astrocytes (using optogenetic tools) induces ATP release and thereby promotes sleep. Loss-of-function studies using a metabolic inhibitor of astrocytes and a pharmacological inhibitor of 
A

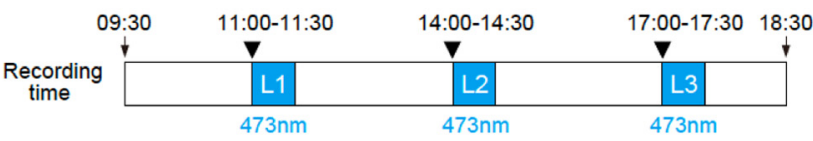

L3 : No Stim

FC EEG

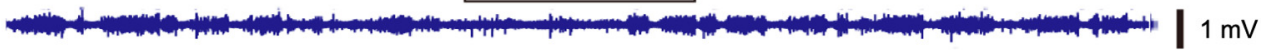

Vibration

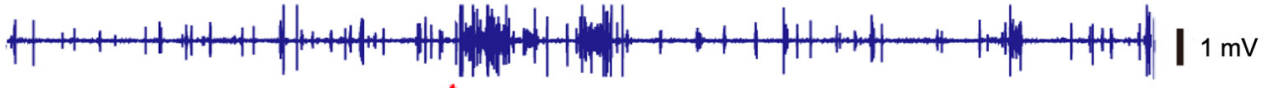

$\Delta$

Hypnogram

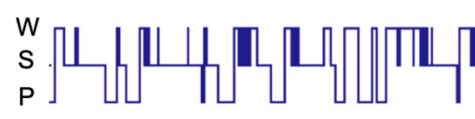

10

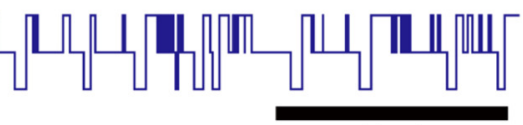

L3 : 473nm

$30 \mathrm{~min}$

FC EEG

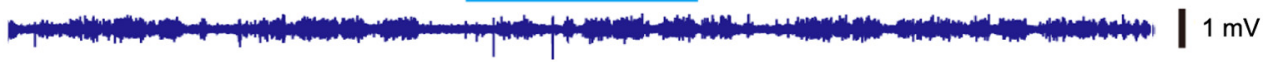

Vibration

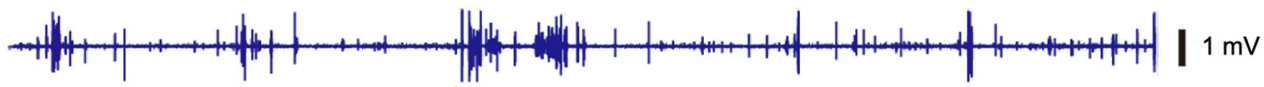

$\Delta$

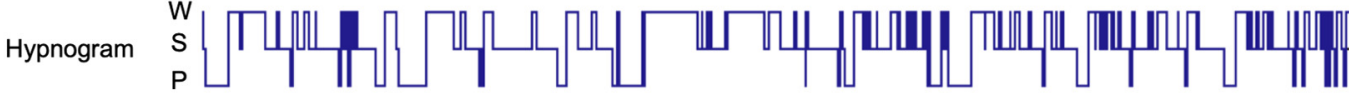

B

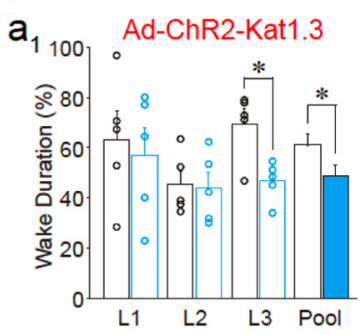

$b_{1100}$

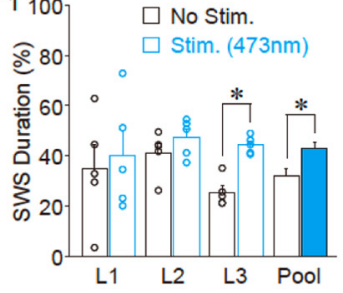

$\mathbf{C}_{1} 100$
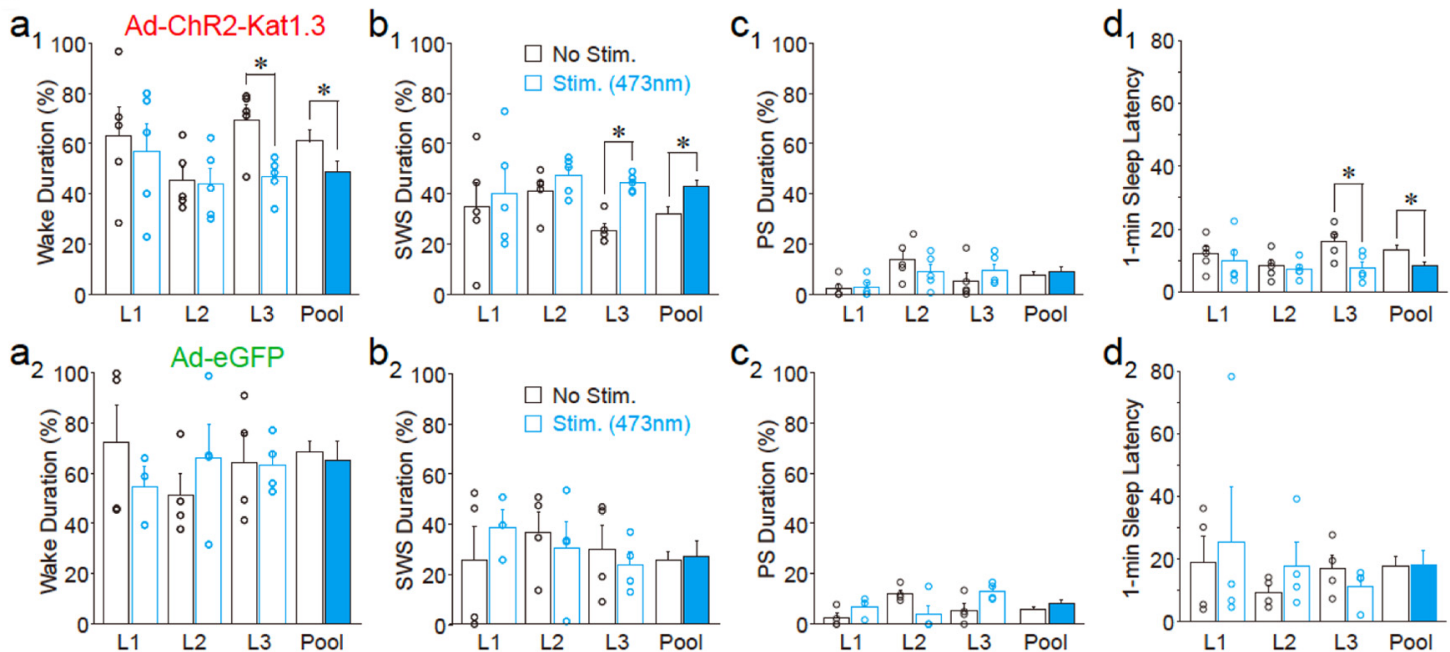

C
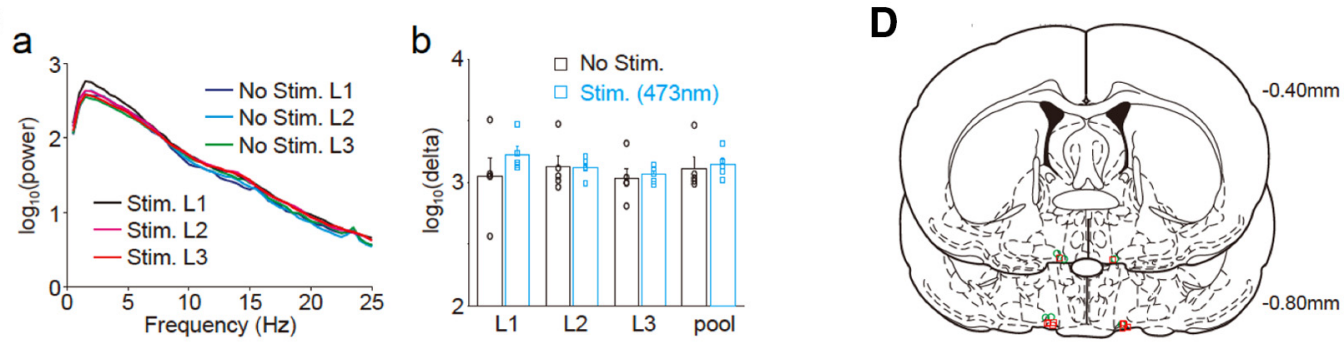

Figure 7. Time-dependent effects of photostimulation on sleep promotion. $A$, Three photostimulation ( $473 \mathrm{~nm}, 1 \mathrm{~Hz}, 500 \mathrm{~ms}$ in duration for 30 min) sessions were conducted at 11 to $11: 30$ A.M. (L1), 2 to 2:30 P.M. (L2), and 5 to 5:30 P.M. (L3) after the awakening (indicated by arrowheads) of rats (top). Representative traces of the frontal cortex EEG (FC EEG), motor activityrelated vibration, and hypnograms (bottom). The recording session included a photostimulation period for $30 \mathrm{~min}$ (L3, blue bar). At the start of each photostimulation, rats were awakened by cage tilting (arrowheads). W, wake; S, SWS; and P, PS. $\boldsymbol{B}$, Photostimulation-induced changes in the duration of wake $\left(\boldsymbol{a}_{\boldsymbol{1}}\right)$, SWS $\left(\boldsymbol{b}_{\boldsymbol{1}}\right)$, PS $\left(\boldsymbol{c}_{1}\right)$, and sleep latency $\left(\boldsymbol{d}_{\boldsymbol{1}}\right)$ in rats with Ad-ChR2 (AdChR2-Kat1.3) expression in the VLP0 area $(n=5)$. Note that photostimulation changed sleep parameters in comparison to those observed in control animals (No Stim, white columns, $n=5$ ) for the $\mathrm{L} 3$, but not for the $\mathrm{L} 1$ or $\mathrm{L} 2$, period. The averaged data for three sessions (pool) are also shown; $* p<0.05$; unpaired $t$ test; wake duration, $\mathrm{L} 3, t_{(8)}=3.33, p=0.0103, \mathrm{pool}, t_{(8)}=$ $2.48, p=0.0378$; SWS duration, $\mathrm{L} 3, t_{(8)}=6.30, p=0.0002$, pool, $t_{(8)}=2.75, p=0.0249$; sleep latency, $\mathrm{L} 3, t_{(8)}=0.0104$, pool, $t_{(8)}=2.675, p=0.0140$. In rats with Ad-eGFP expression in the VLPO area $\left(n=4, \boldsymbol{a}_{2}-\boldsymbol{d}_{2}\right)$, photostimulation (blue columns) did not affect the sleep-wake parameters relative to those of animals that were not photostimulated ( $\left.n=4\right)$. C, EEG power spectra $(\boldsymbol{a})$ and $\delta(0.5-2.5 \mathrm{~Hz})$ power $(\boldsymbol{b})$ during SWS. Power spectra and $\delta$ power were not significantly different among the three sessions regardless of whether they were stimulated or not. All data represent the mean and SEM from five experiments. $\boldsymbol{D}$, Tip locations of the optic cannula in the VLPO area of Ad-ChR2-Kat1.3-injected rats (red squares) and Ad-eGFP-injected rats (green circles) are shown on coronal sections modified from the rat brain atlas. 
A

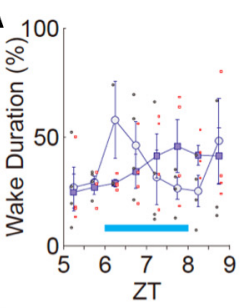

C

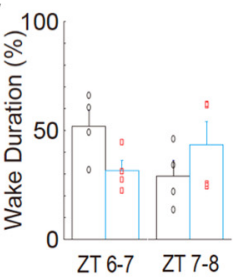

$E_{a}$

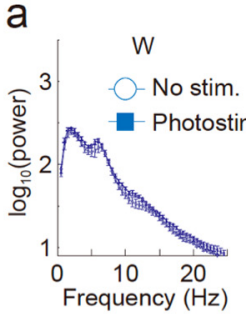

$\mathrm{b}$
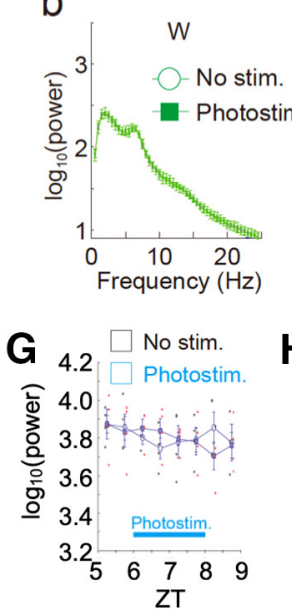

Light period
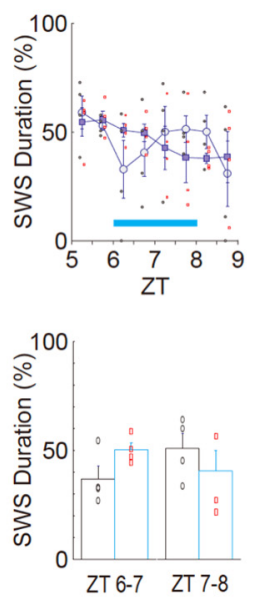

ZT 6-7

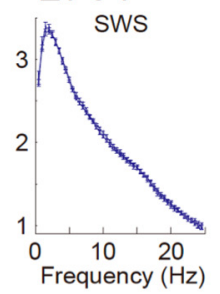

ZT 7-8

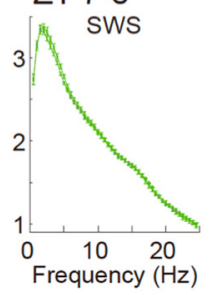

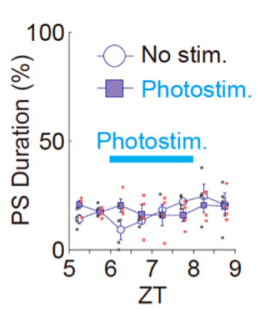
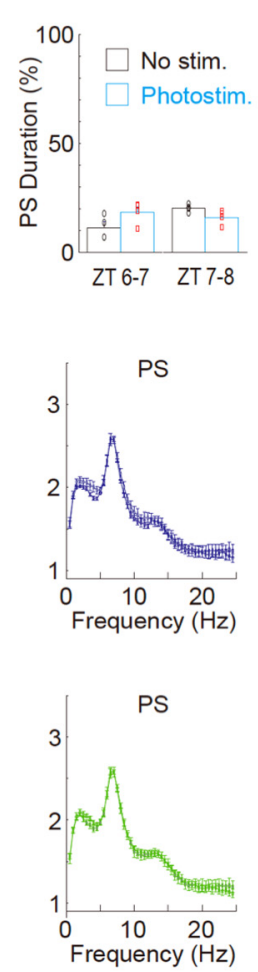

B

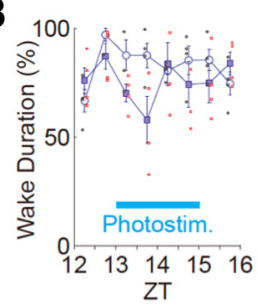

D

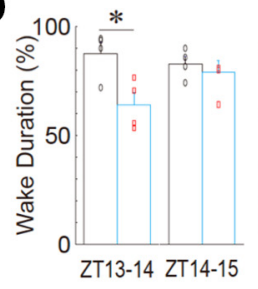

F a

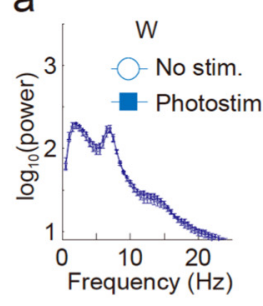

b

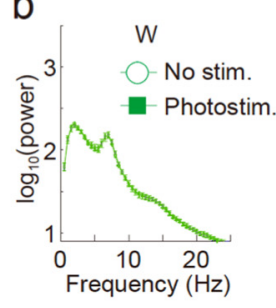

Dark period
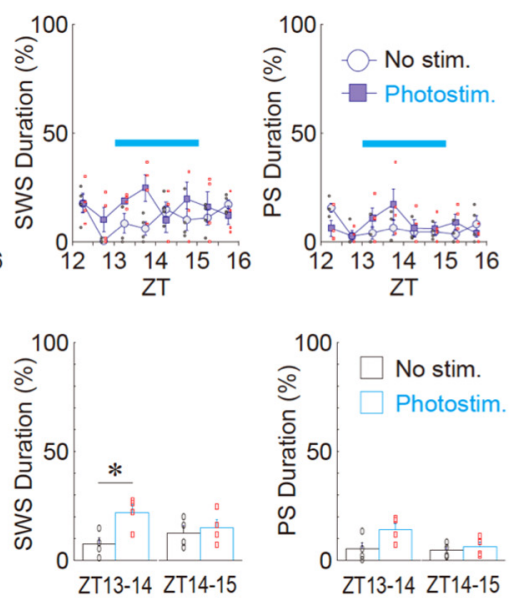

ZT 13-14
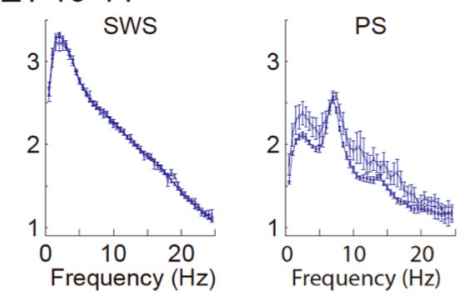

ZT 14-15
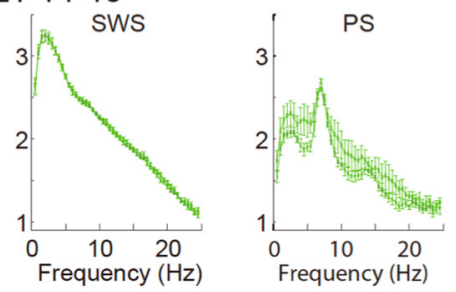
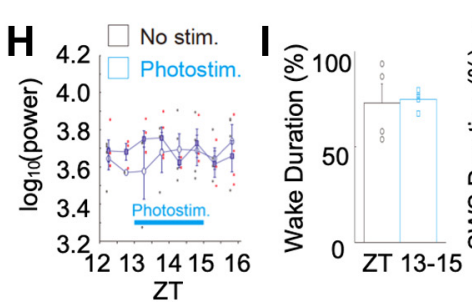

AAV-eYFP (VLPO)

AAV-ChR2-eYFP (Hippocampus)
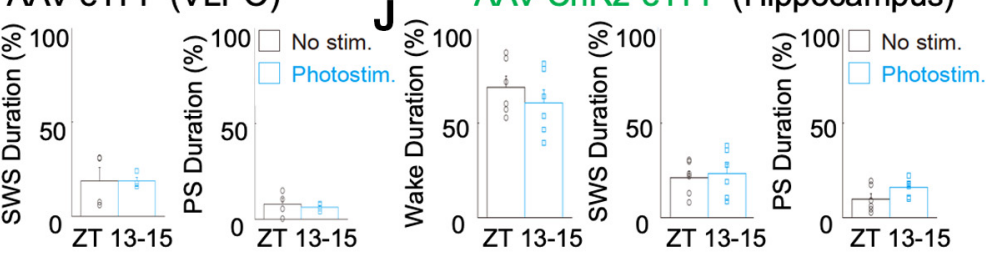

Figure 8. Effects of photostimulation on sleep-wake states and $\mathrm{EEG}$ power in light and dark phases. $\boldsymbol{A}, \boldsymbol{B}$, Changes in the duration of sleep-wake states (wake, SWS, and PS) every 30 min during light $[\boldsymbol{A}$, zeitgeber time (ZT)5-ZT9] and dark (B, ZT12-ZT16) phases. In these experiments, AAV-ChR2-eYFP was delivered to the VLP0 region. Photostimulation was applied to the VLPO region for $120 \mathrm{~min}$ (blue bar). Each point and error bar represents the mean and SEM from four experiments. $\boldsymbol{C}$, $\boldsymbol{D}$, Photostimulation-induced changes in the duration of sleep-wake states during light $(\boldsymbol{C})$ and dark $(\boldsymbol{D})$ phases. Each column and error bar represents the mean and SEM during the first $60 \mathrm{~min}$ and the last $60 \mathrm{~min}$ of photostimulation. Note that in the first $60 \mathrm{~min}$, but not the last $60 \mathrm{~min}$, photostimulation significantly decreased the wake duration and increased the SWS duration during the dark phase $(n=4, D) ; * p<0.05$; unpaired $t$ test; wake duration, ZT13-ZT14, $t_{(6)}=3.02, p=0.0116$; SWS duration, ZT13-ZT14, $t_{(6)}=3.18, p=0.0095$. In contrast, sleep-wake states were not significantly affected by photostimulation during the light phase $(n=4, C) . E, F$, Photostimulation-induced EEG power spectra in sleep-wake states [wake (W), SWS, and PS] during light $(\boldsymbol{E})$ and dark $(\boldsymbol{F})$ phases. Note that there was no significant difference in the power spectra between the unstimulated (No Stim) and photostimulated (Photostim) groups. $\mathbf{G}, \boldsymbol{H}$, Changes in $\delta$ power in the SWS state every 30 min during light (G) and dark (H) phases. Note that there was no significant difference between the two groups. Each point and error bar represents the mean and SEM from four experiments. $I$, Effects of photostimulation on the duration of sleep-wake states (wake, SWS, and PS) during the dark phase. The same photostimulation as that shown in $\boldsymbol{B}$ was applied to the VLPO region, but a control virus without ChR2 (AAV-eYFP) was delivered to the VLPO. Each column and error bar represents the mean and SEM from four experiments. J, Effects of photostimulation on the duration of sleep-wake states (wake, SWS, and PS) during the dark phase. The same 120-min photostimulation period shown in $\boldsymbol{B}$ was applied to the hippocampal region, but AAV-ChR2-eYFP was delivered to the hippocampal CA1 region. Each column and error bar represents the mean and SEM from six experiments. Note that photostimulation of ChR2-expressing astrocytes within the hippocampal region had no influence on sleep-wake states.

TNAP support the critical role of VLPO astrocytes, their release of ATP, and the subsequent hydrolysis of ATP to adenosine in the sleep promotion. In addition to these intervention studies, our observation using histologic and microdialysis analyses that the activity of VLPO astrocytes and ATP levels are increased during sleep states further support the pivotal role of astrocytes and ATP/adenosine in the sleep-promoting VLPO. 
A

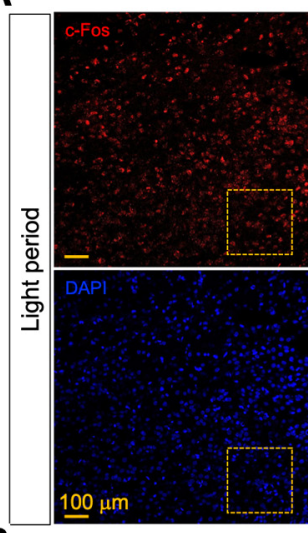

B

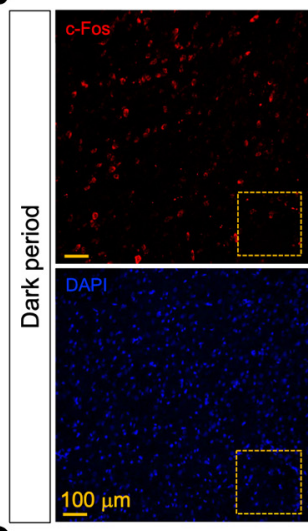

C

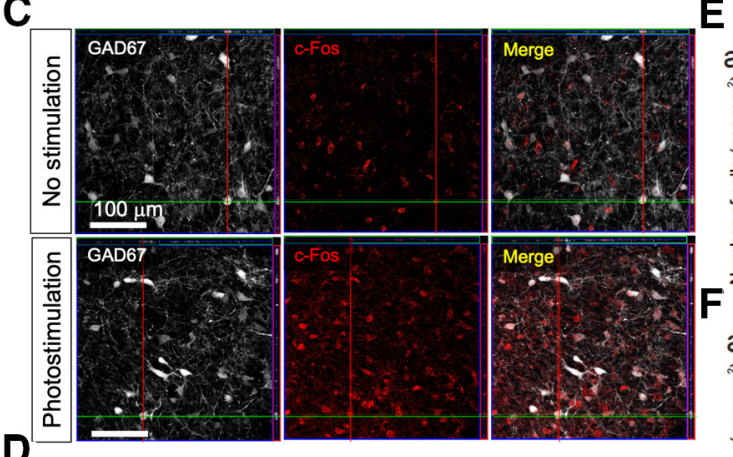

D
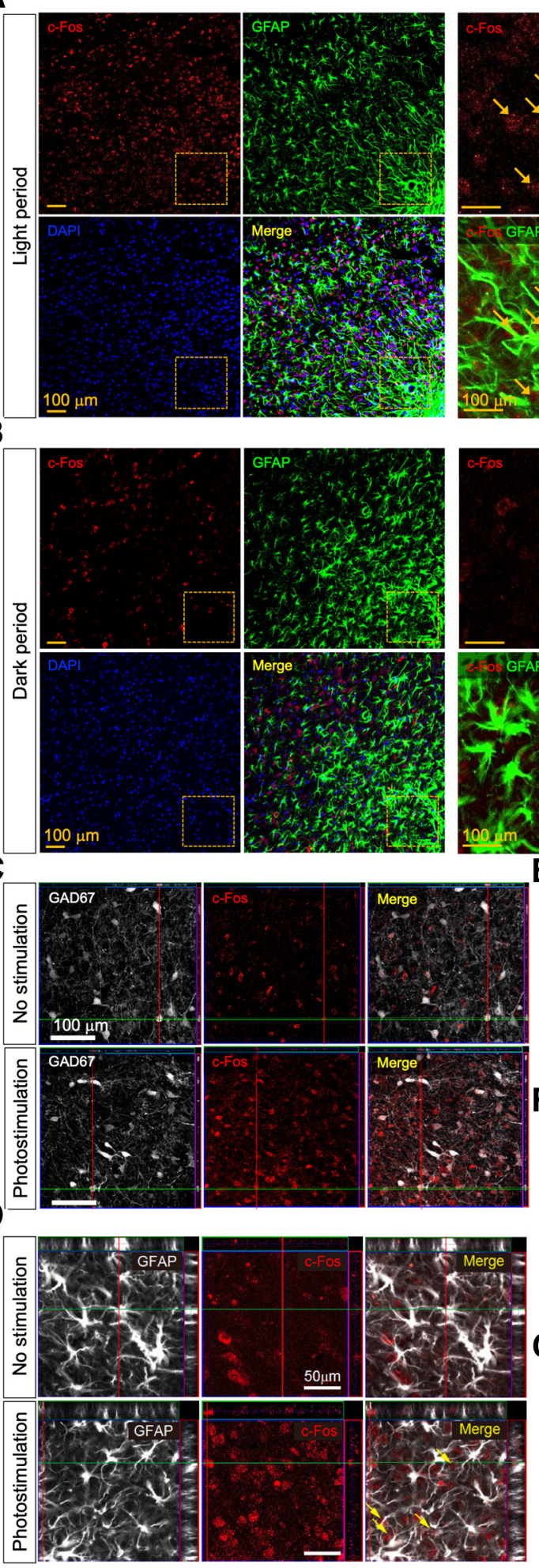

$\mathrm{E}$
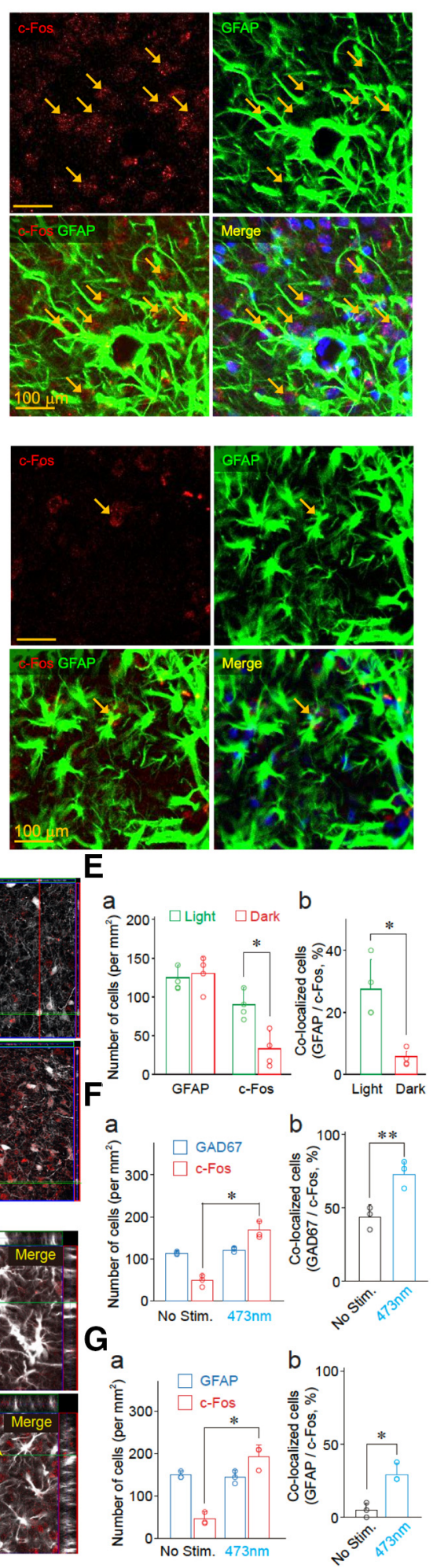

Figure 9. The activity of VLPO astrocytes is increased during sleep states. $A, B, c$-Fos expression in the VLPO astrocytes during the light (sleep; $\boldsymbol{A}$ ) and dark (wake; $\boldsymbol{B}$ ) periods. Confocal images of c-Fos (red) and GFAP immunoreactivity (green) in the VLPO region. Frozen sections of brain were prepared from animals in sleep (light period: 2-4 P.M.) and wake (dark period: 7-9 P.M.) states. Colocalizations of c-Fos (red) and GFAP immunoreactivity (green) in the VLPO region are indicated by arrows. Cell nuclei were stained with DAPI to confirm the nuclear expression of c-Fos. C, D, Increased c-Fos immunoreactivity in GABAergic neurons and astrocytes within the VLPO region following optogenetic stimulation. Rats were injected with AAV-ChR2-eYFP $(n=3)$. Frozen sections of brains were prepared $60 \mathrm{~min}$ after the animals received photostimulation. Neuronal and astrocytic expression of c-Fos in the VLP0 area was identified using anti-GAD67 (C) and anti-GFAP (D) antibodies, respectively, in conjunction with anti-c-Fos antibodies. Cell nuclei were stained with DAPI to confirm the nuclear expression of c-Fos. GAD67-double positive and c-Fos-double positive cells or GFAP-double positive and c-Fos-double positive cells
Glial cells, including astrocytes and microglia, are relevant for sleep regulation during sleep-wake cycle as well as for homeostatic regulation under sleep pressure, as they can affect a variety of neuronal functions by modulating ion buffering, energy metabolism, chemical signaling, synaptic plasticity, and immune system functions (Frank, 2013; Ingiosi et al., 2013; Porkka-Heiskanen, 2013). The first direct evidence for the involvement of brain astrocytes in sleep homeostasis was provided by a previous study in which transgenic animals deficient in astrocyteselective vesicular release were used (Halassa et al., 2009). However, since astrocytic gliotransmission via vesicular release was downregulated in the whole brain in this animal model (Halassa et al., 2009), the roles of astrocytes within specific nuclei involved in sleep regulation were not examined. Pelluru et al. (2016) showed that astrocytes within a specific region such as the posterior hypothalamus are involved in sleep promotion. However, the underlying cellular and molecular mechanisms were not examined in their study. In the present study, we showed an involvement of local gliotransmission as the mechanisms underlying sleep promotion, providing direct evidence that the astrocytes in the VLPO promote sleep.

High levels of c-Fos expression have been detected in the VLPO GABA neurons only during sleeping, but not after

are indicated by arrows. $\boldsymbol{E}$, Quantification of immunopositive cells for GFAP and c-Fos $(\boldsymbol{a})$ and their co-localization $(\boldsymbol{b})$. Frozen sections of brain were prepared from animals in sleep (light period: 2-4 P.M.) and wake (dark period: 7-9 P.M.) states. Astrocytic expression of c-Fos in the VLPO region was identified using an anti-GFAP antibody. Each column and error bar represents the mean and SD from four experiments; $* p<0.05$; unpaired $t$ test; c-Fos positive cells, $t_{(6)}=2.53$, $p=0.0223$; co-localized cells, $t_{(6)}=2.98, p=0.0122 . \boldsymbol{F}$, Quantification of GAD67 and c-Fos immunoreactive cells (a) and their co-localization $(\boldsymbol{b})$. Rats were injected with AAVChR2-eYFP $(n=3)$. Frozen sections of brain were prepared $60 \mathrm{~min}$ after animals received photostimulation. Neuronal expression of C-Fos in the VLPO region was identified using an anti-GAD67 antibody. Each column and error bar represents the mean and SD from three experiments; $* p<0.05$; $* * p<0.01$; unpaired $t$ test; c-Fos-positive cells, $t_{(4)}=2.84$, $p=0.0233$; co-localized cells, $t_{(4)}=3.765, p=0.0098$. $\boldsymbol{G}$, Quantification of immunopositive cells for GFAP and c-Fos $(\boldsymbol{a})$ and their co-localization $(\boldsymbol{b})$. Rats were injected with AAV-ChR2-eYFP $(n=3)$. Frozen sections of brain were prepared $60 \mathrm{~min}$ after the animals received photostimulation. Astrocytic expression of c-Fos in the VLPO region was identified using an anti-GFAP antibody. Each column and error bar represents the mean and SD from three experiments; $* p<0.05$; unpaired $t$ test; $c$-Fos-positive cells, $t_{(4)}=2.96$, $p=0.0206$; co-localized cells, $t_{(4)}=3.54, p=0.0119$. 

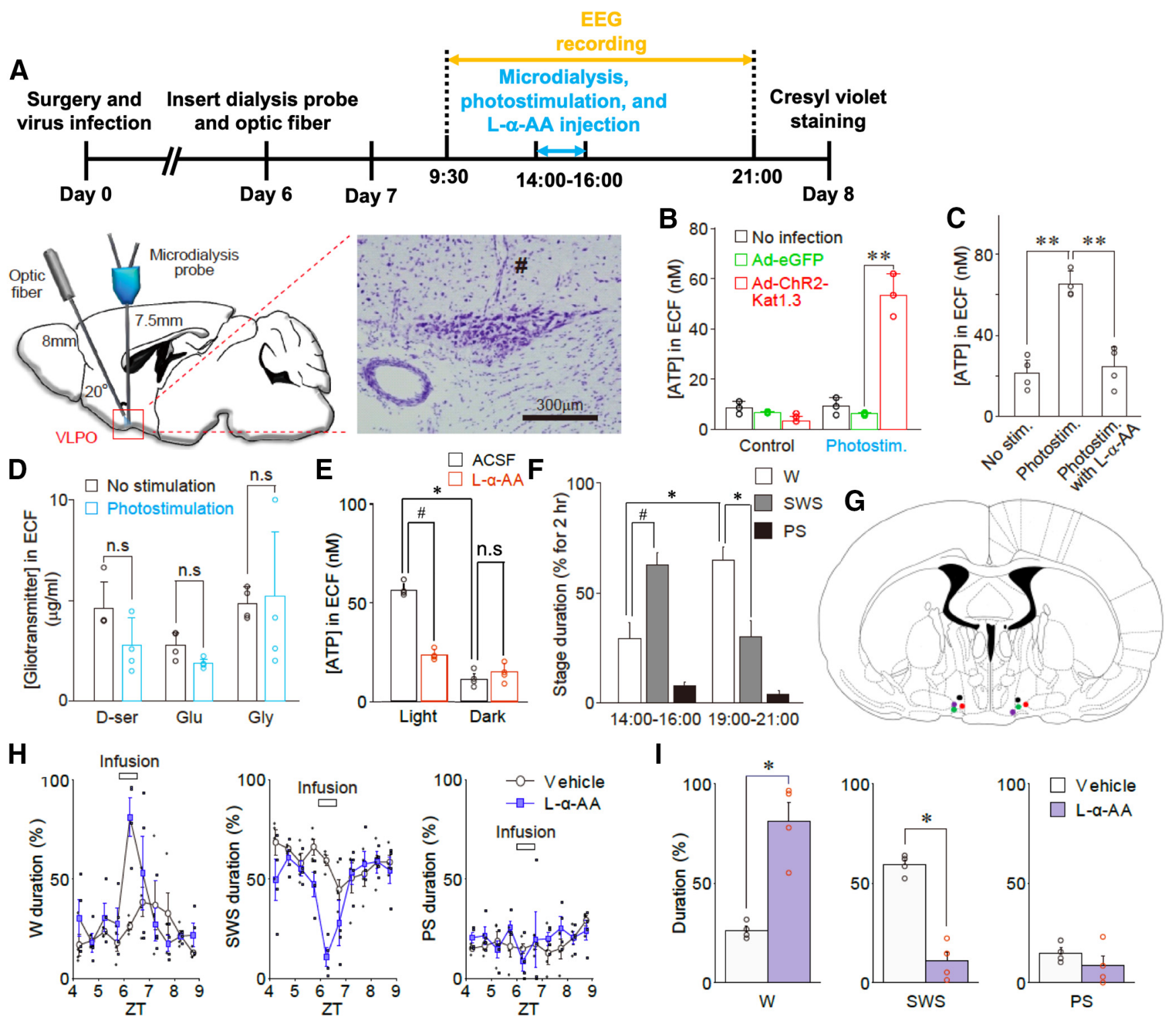

Figure 10. Astocytes release ATP during optogenetic stimulation in vivo. A, Experimental timeline (top) and schematic illustration of microdialysis of the ECFs in the VLPO region during photostimulation (bottom). The microdialysis site was validated by cresyl violet staining at the end of the experiment (right panel); \# represents the insertion site of the microdialysis probe. The results are representative of nine experiments. $\boldsymbol{B}$, The ATP concentration in the dialysate (ECF) before and after photostimulation (120 min). The ATP concentration was measured using a bioluminescence assay. Open circles represent the individual results $(n=3)$, whereas columns and error bars represent the mean and SD from three experiments; $* * p<0.01$; unpaired $t$ test; $t_{(4)}$ $=9.77, p=0.0006$. C, Microdialysis was performed at a flow rate of $0.5 \mathrm{ml} / \mathrm{min}$ with or without photostimulation of the VLPO region for 120 min in the presence or absence of L- $\alpha$-AA (10 nM, a metabolic inhibitor of astrocytes). L- $\alpha$-AA (3 $\mu$ l) was microinjected into the VLPO region 10 min before photostimulation. The concentration of ATP in the VLPO dialysate (ECF) was measured using a bioluminescence assay. Each column and error bar represents the mean and SD from four experiments; $* * p<0.01 ;$ one-way ANOVA; $F_{(2,9)}=40.32, p=0.0001$. $D$, Photostimulation (120 min)-induced changes in D-serine, glutamate, and glycine levels in microdialysates obtained from the VLPO region. Animals were injected with AAV-ChR2-eYFP. The concentrations of D-serine, glutamate, and glycine were determined using an amino acid analyzer; Each column and error bar represents the mean and SD from 4 experiments; n.s., not significant; unpaired $t$ test. $\boldsymbol{E}$, The concentration of ATP in the VLP0 dialysate collected during the light (2-4 P.M.) or dark (7-9 P.M.) period in the presence or absence of L- $\alpha$-AA (10 nM) was measured by a bioluminescence assay. Each column and error bar represents the mean and SD from four experiments; n.s., not significant; $* p<0.05$, paired $t$ test, $t_{(3)}=19.79, p=0.0003$; \#p $<0.05$, paired $t$ test, $t_{(3)}=10.79, p=0.0017$. $F$, The percentage of wake (W), SWS, and PS in the representative wake (dark: 7-9 P.M.) or sleep (light: 2-4 P.M.) period; Each column and error bar represents the mean and SEM from four experiments; $* p<0.05$, paired $t$ test, wake duration, $t_{(3)}=5.01, p=0.0153$; SWS duration, $t_{(3)}=9.43, p=0.0025$; \#p $<0.05$, paired $t$ test, $t_{(3)}=$ $5.53, p=0.0116$. $\mathbf{G}$, A representative image showing each microinjection site of $\mathrm{L}-\alpha$-AA as indicated on the coronal section drawing modified from the rat brain atlas. $\boldsymbol{H}$, Changes in the duration of the sleep-wake states [wake (W), SWS, and PS] at 30-min intervals before, during, and after treatment with saline or L- $\alpha$-AA (horizontal bar). Saline or L- $\alpha$-AA (3 $\mu$ l, $10 \mathrm{~nm})$ was directly microinjected into the VLPO region for $30 \mathrm{~min}$ at a rate of $0.1 \mu \mathrm{l} / \mathrm{min}$. Each column and error bar represents the mean and SEM from four experiments; $\boldsymbol{I}$, The duration of the sleepwake states during microinjection of saline or L- $\alpha$-AA. Each column and error bar represents the mean and SEM from four experiments; $* p<0.05$; unpaired $t$ test; W duration, $t_{(6)}=5.57$, $p=0.0014$; SWS duration, $t_{(6)}=9.02, p=0.0001$.

sleep deprivation (Sherin et al., 1996). Electrophysiological recording has also supported that most neurons fire during sleep (Szymusiak et al., 1998). The c-Fos expression as a histologic activity marker and electrophysiological recording data support that the VLPO sleep-active neurons are involved in sleep maintenance rather than sleep induction (Strecker et al., 2000). In addition, levels of c-Fos expression in VLPO astrocytes were higher in light phase than in dark phase (Fig. 9), which was consistent with a causal relationship between VLPO astrocytes and sleep. In agreement with a previous report of an increase in c-Fos 
A

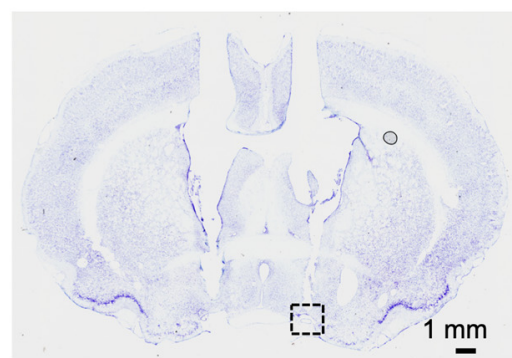

B

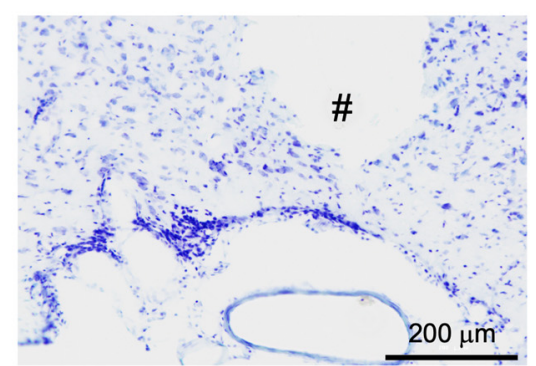

Figure 11. The microdialysis site was validated by cresyl violet staining at the end of the experiment (coronal section; $\boldsymbol{A}$ ); \# represents the insertion site of the microdialysis probe in high-magnification image $(\boldsymbol{B})$.
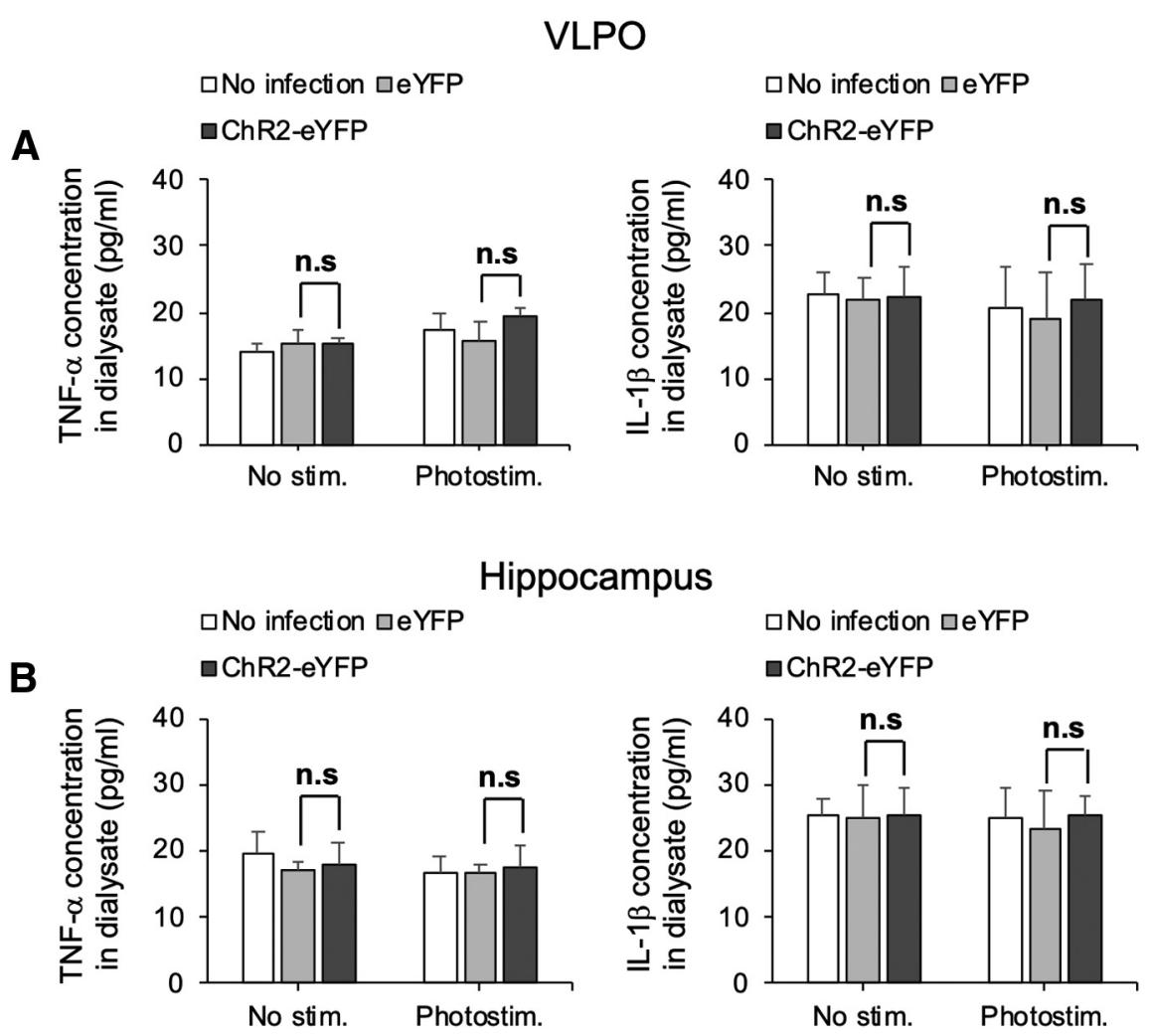

Figure 12. Cytokine levels in the dialysate (ECF) before and after photostimulation (120 min). TNF- $\alpha$ and IL-1 $\beta$ levels were measured using ELISA assay in VLPO $(\boldsymbol{A})$ and hippocampus $(\boldsymbol{B})$. Columns and error bars represent the mean and SD from four experiments; n.s., not significant; one-way ANOVA.

expression in VLPO neurons during sleep (Sherin et al., 1996), we also observed that c-Fos expressions increased in VLPO neurons as well as VLPO astrocytes, when VLPO astrocytes were stimulated by optogenetic tools (Fig. 9). These results led us to hypothesize that the activated astrocytes in VLPO release gliotransmitters, which in turn promote sleep. Nevertheless, c-Fos expression, as an indirect histologic activity marker, has limitations. Further studies are necessary to definitively demonstrate the astrocyte activities in VLPO with respect to sleep regulation.

Astrocytes are known to release gliotransmitters that can modulate the excitability of adjacent neurons. Several gliotransmitters, including ATP, glutamate, and GABA, have been identified in various brain regions (Gourine et al., 2010; Araque et al., 2014). Among these, ATP can be released from astrocytes via vesicle fusion (Pascual et al., 2005) or via several ion channels, including hemichannels and P2X7 receptors (Lohman and
Isakson, 2014; Montero and Orellana, 2015) in response to glutamate (Porter and McCarthy, 1996; Wang et al., 2006; Barat et al., 2012) or GABA (Serrano et al., 2006). Given that adenosine, a by-product of energy metabolism, is regarded as an endogenous substance that can promote sleep (Basheer et al., 2004; Huang et al., 2005) and that ATP can easily be degraded into adenosine by several enzymes (Dunwiddie et al., 1997; Cunha et al., 1998; Zimmermann, 2000; Zylka et al., 2008; Street et al., 2013), we hypothesized that ATP acts as a gliotransmitter within the VLPO region. Indeed, by using a microdialysis technique, we found that ATP was released in living animals during optogenetic stimulation of VLPO astrocytes (Fig. 10). We also found that the ATP concentration within the VLPO region was higher during light phase than during dark phase, and we observed that a metabolic inhibitor of astrocytes significantly reduced extracellular ATP levels and sleep duration (Fig. 10); thus, ATP released from VLPO astrocytes apparently plays a pivotal role in sleep promotion. Although our in vitro experiments support the conclusion that ATP is released from astrocytes through, at least in part, hemichannels and/or $\mathrm{P} 2 \mathrm{X} 7$ receptors (Fig. 13), the possibility that ATP is released from astrocytes via vesicular fusion cannot be excluded. In addition, it is possible that the photostimulation of ChR2-expressing astrocytes used in the present study differs from the physiological stimuli that activate astrocytes within the brain. Nonetheless, we found that ChR2 (H134R) shows a small $\mathrm{Ca}^{2+}$ permeability in cultured astrocytes (Fig. 1D), and that photostimulation evokes an increase in intracellular $\mathrm{Ca}^{2+}$ concentration as well as its sustained oscillation (Fig. 1E-I). Thus, our experimental method of using ChR2 (Ad-ChR2-Kat1.3 or AAV-ChR2-eYFP) appears to drive $\mathrm{Ca}^{2+}$ responses in ChR2-expressing astrocytes. Such $\mathrm{Ca}^{2+}$ responses, which were elicited by the activation of G-protein coupled receptors in native astrocytes (Perea and Araque, 2005; Panatier et al., 2011), successfully trigger the release of gliotransmitters (Bezzi and Volterra, 2001; Perea et al., 2014).

We found that photostimulation of the VLPO astrocytes promoted sleep only when the rats were mostly awake but not when they were mostly asleep. This indicates that the VLPO astrocytes play an important role in sleep regulation. Similarly, photostimulation of the astrocytes in the posterior hypothalamus also increased sleep when the rats were mostly awake (Pelluru et al., 2016). Furthermore, recent studies have indicated an important role of cortical astrocytes in the sleep regulation (Bojarskaite et al., 2020; Ingiosi 
A

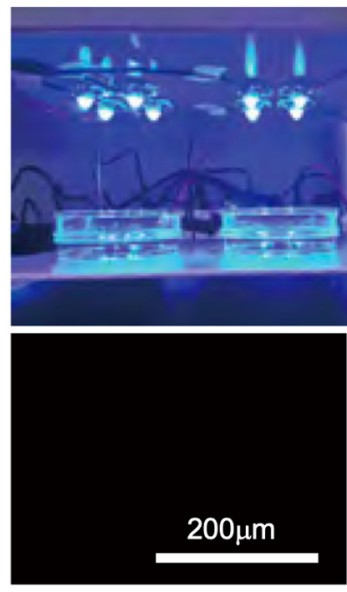

No infection
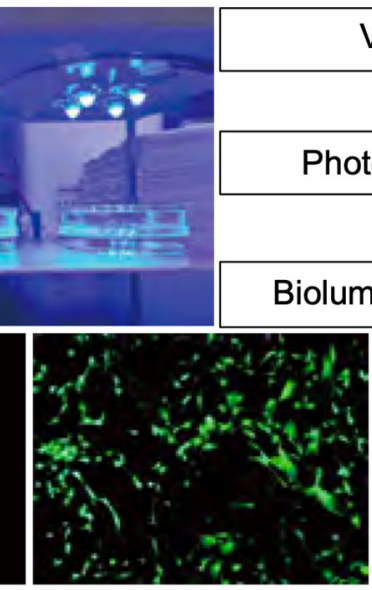

Ad-EGFP

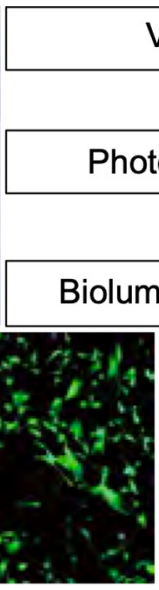

B

* D

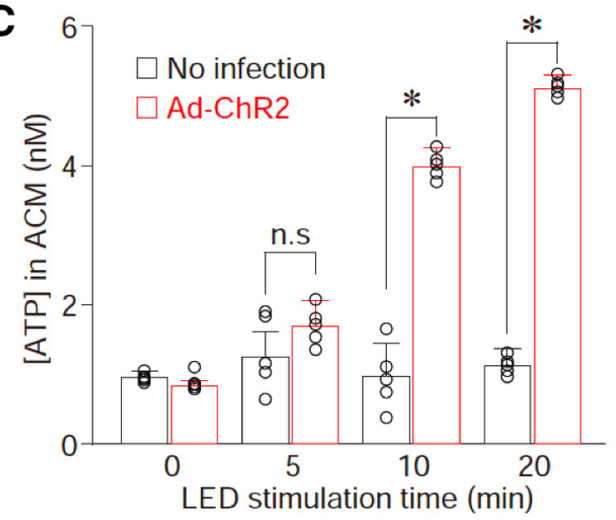

D

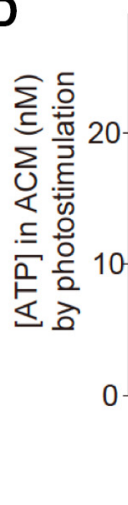

Control

Photostimulation

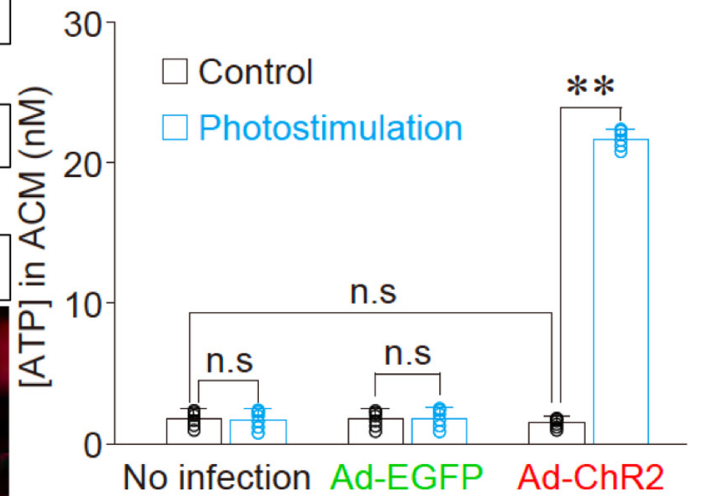

Figure 13. Optogenetic stimulation of cultured astrocytes induces ATP release. A, Primary astrocytes were infected with either Ad-EGFP (control virus, green, $7.6 \times 1010$ $\mathrm{PFU} / \mathrm{ml}$ ) or Ad-ChR2 (red). Two days after virus infection, cultured astrocytes were optically stimulated using an LED device before bioluminescence analysis of ATP release. The results are representative of three to six experiments. $\boldsymbol{B}$, The extracellular ATP concentration was measured from the astrocyte-conditioned medium (ACM) in cultured astrocytes infected with either Ad-EGFP or Ad-ChR2 after 2-h photostimulation. Open circles represent the individual results $(n=6)$, and columns and error bars represent the mean and SD from six experiments; n.s., not significant; $* * p<0.01$; unpaired $t$ test, $t_{(10)}=29.50, p=0.0001$. C, The extracellular ATP concentration was measured from the ACM in cultured astrocytes infected with Ad-ChR2 by various duration of photostimulation. Open circles represent the individual results $(n=6)$, and columns and error bars represent the mean and SD from six experiments; n.s., not significant; $* * p<0.01$; unpaired $t$ test, 10 min, $t_{(10)}=8.67, p=0.0001$; $20 \mathrm{~min}, t_{(10)}=15.88, p=0.0001$. D, Effects of Brilliant Blue G (BBG, a P2X7 receptor antagonist, $100 \mathrm{~nm}$ ) or (BX (a hemichannel blocker, $3 \mu \mathrm{m}$ ) on ATP release in cultured astrocytes. ChR2-expressing astrocytes were illuminated with LED in the absence (vehicle) or presence of $100 \mathrm{~nm} \mathrm{BBG}$ or $3 \mu \mathrm{m}$ CBX for $2 \mathrm{~h}$. Extracellular ATP concentration was measured by a bioluminescence assay. Open circles represent the individual results $(n=5)$, and columns and error bars represent the mean and SD from five experiments; $* * p<0.01$; one-way ANOVA, $F_{(2,12)}=29.42, p=0.0001$. $E$, Cell viability was measured by MTT assays $24 \mathrm{~h}$ after the treatment of $100 \mathrm{~nm} B B G$ or $3 \mu \mathrm{m}$ CBX. Open circles represent the individual results $(n=4)$, and columns and error bars represent the mean and SD from four experiments; n.s.; not significant; one-way ANOVA.

et al., 2020). Therefore, sleep may be regulated by cooperation of astrocytes in the multiple sleep-wake regulatory regions within the brain. Whether astrocytes in other brain regions, such as basal forebrain and brain stem, also participate in sleep regulation remains to be studied.

Astrocytes are a major source of ATP, which is converted to adenosine. We found that the release of ATP from VLPO astrocytes is increased during natural sleep and also by photostimulation of the astrocytes. Genetically engineered mice with reduced vesicular ATP release from astrocytes have normal sleep-wake profiles but do not show a sleep rebound in response to sleep deprivation or blocking of A1 receptors (Halassa et al., 2009). Although systemic or intracerebroventricular administration of adenosine promotes sleep (Radulovacki et al., 1984; Ticho and Radulovacki, 1991), microinjection of adenosine and A1 receptor agonist into the VLPO decreases sleep (Zhang et al., 2013). In vitro studies show that A1 receptor agonists block VLPO inhibitory interneurons (Morairty et al., 2004). A2a receptor agonists directly excite some VLPO sleep-promoting neurons (Gallopin et al., 2005). Also, A1 receptors, but not $\mathrm{A} 2 \mathrm{a}$ receptors, are expressed in the VLPO (Dixon et al., 1996). Thus, it was hypothesized that the action through A1 receptor, but not A2a receptor, may inhibit the VLPO interneurons, which disinhibit VLPO sleep-promoting neurons. Indeed, a recent study has reported that selective activation of VLPO glalaninergic neurons increases sleep (Kroeger et al., 2018). Therefore, we postulate that ATP released from the VLPO astrocytes may inhibit the VLPO neurons via A1 receptors, and then disinhibits VLPO sleep-promoting neurons, thereby increasing sleep. How adenosine selectively acts on the GABAergic interneurons, but not on the GABAergic projection neurons intermingled in the VLPO, remains to be investigated.

Extracellular adenosine accumulates within the basal forebrain and cortex, but not other brain regions, during sleep deprivation (Porkka-Heiskanen et al., 1997; Strecker et al., 2000). 


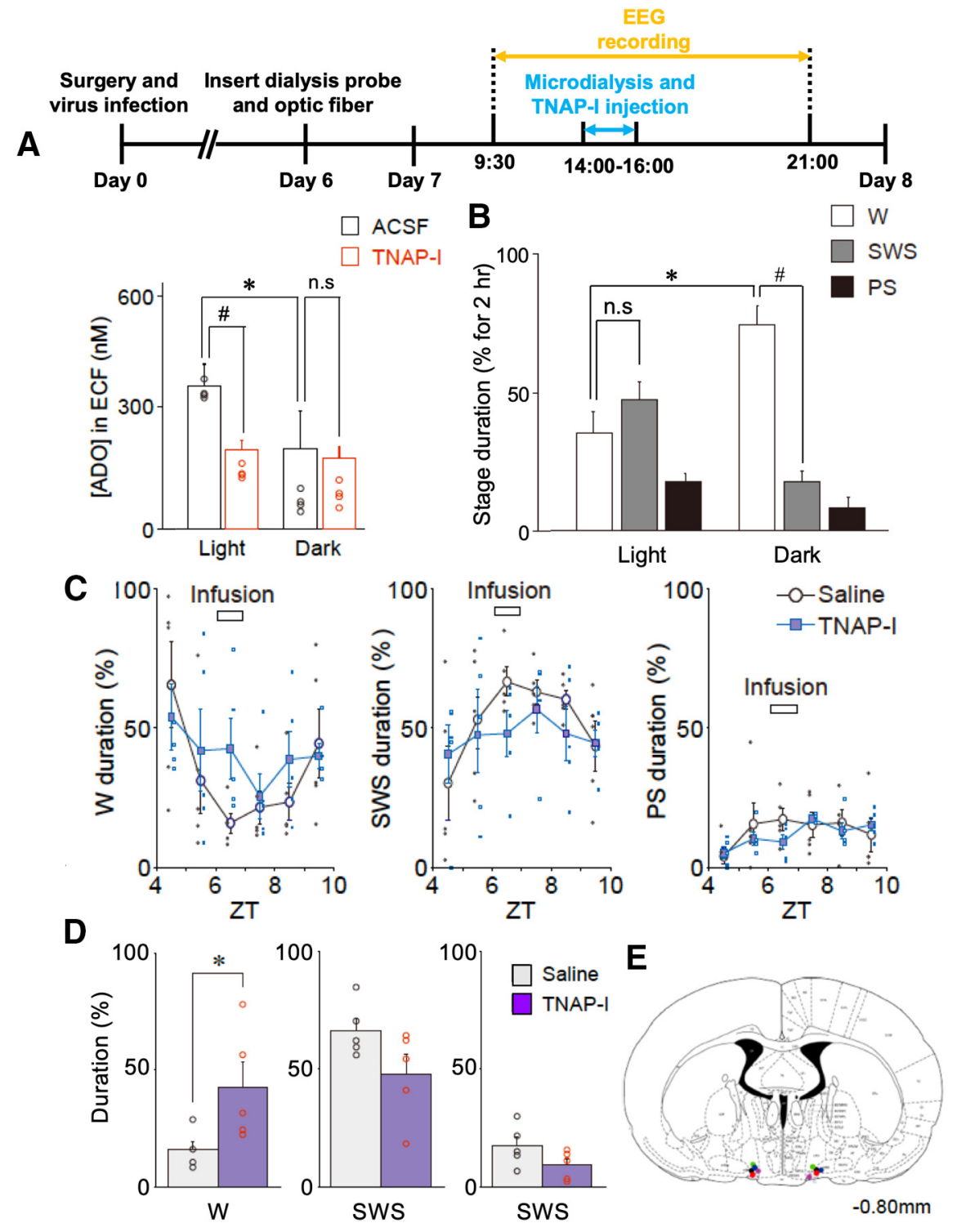

Figure 14. TNAP plays pivotal roles in sleep-wake states. $\boldsymbol{A}$, Experimental timeline (top) and the concentration of adenosine (AD0) in the VLPO dialysate collected during the light (2-4 P.M.) or dark (7-9 P.M.) period in the presence or absence of TNAP-I was measured by HPLC assay. ACSF or TNAP-I $(3 \mu \mathrm{l}, 10 \mathrm{mg} / \mathrm{ml})$ was directly microinjected into the VLP0 region for $30 \mathrm{~min}$ at a rate of $0.1 \mu \mathrm{l} / \mathrm{min}$. The results are representative of four experiments; n.S., not significant; $* p<0.05$, paired $t$ test, $t_{(4)}=3.19, p=0.0331 ; \# p<0.05$, unpaired $t$ test, $t_{(4)}=5.79, p=0.0044$. $\boldsymbol{B}$, The percentage of wake (W), SWS, and PS in the representative wake (dark: 7-9 P.M.) or sleep (light: 2-4 P.M.) periods; Each column and error bar represents the mean and SEM from four experiments; n.S., not significant; $* p<0.05$; paired $t$ test, $t_{(3)}=9.13, p=0.0028$; \#p $<0.05$; unpaired $t$ test, $t_{(6)}=13.50, p=0.0001$. $C$, Changes in the duration of the sleep-wake states [wake (W), SWS, and PS] at 30-min intervals before, during, and after treatment with saline or TNAP-I (horizontal bar). Saline or TNAP-I (3 $\mu$ l, $10 \mathrm{mg} /$ $\mathrm{ml}$ ) was directly microinjected into the VLPO region for $30 \mathrm{~min}$ at a rate of $0.1 \mu \mathrm{l} / \mathrm{min}$. Each column and error bar represents the mean and SEM from four experiments. $\boldsymbol{D}$, The duration of the sleep-wake states during microinjection of saline or TNAPI. Each column and error bar represents the mean and SEM from five experiments; $* p<0.05$; unpaired $t$ test, $t_{(8)}=2.34$, $p=0.0473$. Note that the duration of the wake state was significantly increased in the TNAP-I group. $\boldsymbol{E}$, The image represents each microinjection site of TNAP-I shown on coronal sections modified from the rat brain atlas.

Adenosine levels were evaluated in all brain regions (including the cortex) of cat (Porkka-Heiskanen et al., 2000) and in the basal forebrain (Murillo-Rodriguez et al., 2004) and the hippocampus (Huston et al., 1996) of rat: there were variations in adenosine levels and metabolizing enzymes depending on the sleep-wake cycle (Chagoya de Sánchez et al., 1993). Our results show that extracellular levels of both ATP and adenosine in the VLPO are higher during spontaneous sleep (light phase) than during wake (dark phase). This discrepancy in adenosine levels among the previous studies may be because of differences in species or brain regions. Indeed, adenosine level is regulated by complex interactions of production and degradation enzymes as well as equilibrative nucleoside transporters in the astrocytes (Strecker et al., 2000). Activities of metabolic enzymes producing or degrading adenosine show diurnal rhythms (Chagoya de Sánchez et al., 1993; Mackiewicz et al., 2003) and their distributions are different among brain regions (Mackiewicz et al., 2003). Stimulation of the posterior hypothalamus astrocytes induced a progressive increase in sleep duration in the first $4 \mathrm{~h}$ and a decrease in the subsequent $2 \mathrm{~h}$ during a total 6-h stimulation period (Pelluru et al., 2016). It was suggested that the cumulative effect may result from the gradual accumulation of gliotransmitters, such as adenosine. We also observed a gradual increase in sleep duration when three successive stimulations were given along a single day (Fig. 7). However, we could not observe the progressive increase across a 2 -h stimulation session (Fig. 8). This may be because of the relative short duration of stimulation; however, this possibility needs to be tested further. Another possibility is a difference in the adenosine clearance rate among distinct brain regions, where distribution and diurnal variation of the metabolic enzymes are different (Mackiewicz et al., 2003). For example, activity of adenosine deaminase during the active period (night) is decreased in the basal forebrain and the posterior hypothalamus, while it is increased in the VLPO. Thus, adenosine derived from the astrocytes may be rapidly metabolized as soon as it acts on nearby neurons in VLPO.

At this stage, the physiological signals responsible for the increase in endogenous local adenosine within the VLPO region, which might be because of the hydrolysis of ATP released from astrocytes under conditions related to sleep promotion, remain to be elucidated. It is possible that the inputs from reciprocal inhibitory neural circuits involved in sleep-wake regulation, such as the wake-promoting nuclei in the brain stem, the lateral hypothalamus, and the median preoptic area (Chou et al., 2002), are also involved in the astrocytic ATP release and subsequent degradation to adenosine; this speculation also requires further investigation.

In conclusion, we have shown that astrocytes within the VLPO region promote sleep via ATP release and the subsequent hydrolysis by an ATP-degrading enzyme TNAP. Our findings suggest a new glio-centric mechanism of sleep regulation, which takes place in the VLPO in cooperation with other brain regions related to sleep-wake regulation. 


\section{References}

Araque A, Carmignoto G, Haydon PG, Oliet SH, Robitaille R, Volterra A (2014) Gliotransmitters travel in time and space. Neuron 81:728-739.

Barat E, Boisseau S, Bouyssières C, Appaix F, Savasta M, Albrieux M (2012) Subthalamic nucleus electrical stimulation modulates calcium activity of nigral astrocytes. PLoS One 7:e41793.

Basheer R, Strecker RE, Thakkar MM, McCarley RW (2004) Adenosine and sleep-wake regulation. Prog Neurobiol 73:379-396.

Ben Achour S, Pascual O (2012) Astrocyte-neuron communication: functional consequences. Neurochem Res 37:2464-2473.

Benington JH, Heller HC (1995) Restoration of brain energy metabolism as the function of sleep. Prog Neurobiol 45:347-360.

Bennett MV, Contreras JE, Bukauskas FF, Sáez JC (2003) New roles for astrocytes: gap junction hemichannels have something to communicate. Trends Neurosci 26:610-617.

Bezzi P, Volterra A (2001) A neuron-glia signalling network in the active brain. Curr Opin Neurobiol 11:387-394.

Bianco F, Pravettoni E, Colombo A, Schenk U, Möller T, Matteoli M, Verderio C (2005) Astrocyte-derived ATP induces vesicle shedding and IL-1 beta release from microglia. J Immunol 174:7268-7277.

Bojarskaite L, Bjørnstad DM, Pettersen KH, Cunen C, Hermansen GH, Åbjørsbråten KS, Chambers AR, Sprengel R, Vervaeke K, Tang W, Enger $\mathrm{R}$, Nagelhus EA (2020) Astrocytic $\mathrm{Ca}(2+)$ signaling is reduced during sleep and is involved in the regulation of slow wave sleep. Nat Commun 11:3240.

Borbély AA, Daan S, Wirz-Justice A, Deboer T (2016) The two-process model of sleep regulation: a reappraisal. J Sleep Res 25:131-143.

Chagoya de Sánchez V, Hernández Múñoz R, Suárez J, Vidrio S, Yáñez L, Díaz Múñoz M (1993) Day-night variations of adenosine and its metabolizing enzymes in the brain cortex of the rat-possible physiological significance for the energetic homeostasis and the sleep-wake cycle. Brain Res 612:115-121.

Chou TC, Bjorkum AA, Gaus SE, Lu J, Scammell TE, Saper CB (2002) Afferents to the ventrolateral preoptic nucleus. J Neurosci 22:977-990.

Choudhury SR, Harris AF, Cabral DJ, Keeler AM, Sapp E, Ferreira JS, GrayEdwards HL, Johnson JA, Johnson AK, Su Q, Stoica L, DiFiglia M, Aronin N, Martin DR, Gao G, Sena-Esteves M (2016) Widespread central nervous system gene transfer and silencing after systemic delivery of novel AAV-AS vector. Mol Ther 24:726-735.

Cunha RA, Sebastião AM, Ribeiro JA (1998) Inhibition by ATP of hippocampal synaptic transmission requires localized extracellular catabolism by ecto-nucleotidases into adenosine and channeling to adenosine A1 receptors. J Neurosci 18:1987-1995.

Dahl R, Sergienko EA, Su Y, Mostofi YS, Yang L, Simao AM, Narisawa S, Brown B, Mangravita-Novo A, Vicchiarelli M, Smith LH, O'Neill WC, Millán JL, Cosford NDP (2009) Discovery and validation of a series of aryl sulfonamides as selective inhibitors of tissue-nonspecific alkaline phosphatase (TNAP). J Med Chem 52:6919-6925.

Dixon AK, Gubitz AK, Sirinathsinghji DJ, Richardson PJ, Freeman TC (1996) Tissue distribution of adenosine receptor mRNAs in the rat. Br J Pharmacol 118:1461-1468.

Dunwiddie TV, Diao L, Proctor WR (1997) Adenine nucleotides undergo rapid, quantitative conversion to adenosine in the extracellular space in rat hippocampus. J Neurosci 17:7673-7682.

Faul F, Erdfelder E, Buchner A, Lang AG (2009) Statistical power analyses using $\mathrm{G} *$ Power 3.1: tests for correlation and regression analyses. Behav Res Methods 41:1149-1160.

Figueiredo M, Lane S, Tang F, Liu BH, Hewinson J, Marina N, Kasymov V, Souslova EA, Chudakov DM, Gourine AV, Teschemacher AG, Kasparov S (2011) Optogenetic experimentation on astrocytes. Exp Physiol 96:4050 .

Frank MG (2013) Astroglial regulation of sleep homeostasis. Curr Opin Neurobiol 23:812-818.

Fujii Y, Maekawa S, Morita M (2017) Astrocyte calcium waves propagate proximally by gap junction and distally by extracellular diffusion of ATP released from volume-regulated anion channels. Sci Rep 7:13115.

Gallopin T, Fort P, Eggermann E, Cauli B, Luppi PH, Rossier J, Audinat E, Mühlethaler M, Serafin M (2000) Identification of sleep-promoting neurons in vitro. Nature 404:992-995.

Gallopin T, Luppi PH, Cauli B, Urade Y, Rossier J, Hayaishi O, Lambolez B, Fort P (2005) The endogenous somnogen adenosine excites a subset of sleep-promoting neurons via $\mathrm{A} 2 \mathrm{~A}$ receptors in the ventrolateral preoptic nucleus. Neuroscience 134:1377-1390.

Gandelman M, Peluffo H, Beckman JS, Cassina P, Barbeito L (2010) Extracellular ATP and the P2X7 receptor in astrocyte-mediated motor neuron death: implications for amyotrophic lateral sclerosis. J Neuroinflammation 7:33.

Gourine AV, Kasymov V, Marina N, Tang F, Figueiredo MF, Lane S, Teschemacher AG, Spyer KM, Deisseroth K, Kasparov S (2010) Astrocytes control breathing through $\mathrm{pH}$-dependent release of ATP. Science 329:571-575.

Grafe LA, Takacs AE, Yee DK, Flanagan-Cato LM (2014) The role of the hypothalamic paraventricular nucleus and the organum vasculosum lateral terminalis in the control of sodium appetite in male rats. J Neurosci 34:9249-9260.

Guenthner CJ, Miyamichi K, Yang HH, Heller HC, Luo L (2013) Permanent genetic access to transiently active neurons via TRAP: targeted recombination in active populations. Neuron 78:773-784.

Guo Z, Zhang L, Wu Z, Chen Y, Wang F, Chen G (2014) In vivo direct reprogramming of reactive glial cells into functional neurons after brain injury and in an Alzheimer's disease model. Cell Stem Cell 14:188-202.

Halassa MM, Florian C, Fellin T, Munoz JR, Lee SY, Abel T, Haydon PG, Frank MG (2009) Astrocytic modulation of sleep homeostasis and cognitive consequences of sleep loss. Neuron 61:213-219.

Hide I, Tanaka M, Inoue A, Nakajima K, Kohsaka S, Inoue K, Nakata Y (2000) Extracellular ATP triggers tumor necrosis factor-alpha release from rat microglia. J Neurochem 75:965-972.

Huang ZL, Qu WM, Eguchi N, Chen JF, Schwarzschild MA, Fredholm BB, Urade Y, Hayaishi O (2005) Adenosine A2A, but not A1, receptors mediate the arousal effect of caffeine. Nat Neurosci 8:858-859.

Huston JP, Haas HL, Boix F, Pfister M, Decking U, Schrader J, Schwarting RK (1996) Extracellular adenosine levels in neostriatum and hippocampus during rest and activity periods of rats. Neuroscience 73:99-107.

Ingiosi AM, Opp MR, Krueger JM (2013) Sleep and immune function: glial contributions and consequences of aging. Curr Opin Neurobiol 23:806811.

Ingiosi AM, Hayworth CR, Harvey DO, Singletary KG, Rempe MJ, Wisor JP, Frank MG (2020) A role for astroglial calcium in mammalian sleep and sleep regulation. Curr Biol. Available at https://doi. org/10.1016/j.cub.2020.08.052

Jha MK, Song GJ, Lee MG, Jeoung NH, Go Y, Harris RA, Park DH, Kook H, Lee IK, Suk K (2015) Metabolic connection of inflammatory pain: pivotal role of a pyruvate dehydrogenase kinase-pyruvate dehydrogenase-lactic acid axis. J Neurosci 35:14353-14369.

Jiang BC, Cao DL, Zhang X, Zhang ZJ, He LN, Li CH, Zhang WW, Wu XB, Berta T, Ji RR, Gao YJ (2016) CXCL13 drives spinal astrocyte activation and neuropathic pain via CXCR5. J Clin Invest 126:745-761.

Kroeger D, Absi G, Gagliardi C, Bandaru SS, Madara JC, Ferrari LL, Arrigoni E, Münzberg H, Scammell TE, Saper CB, Vetrivelan R (2018) Galanin neurons in the ventrolateral preoptic area promote sleep and heat loss in mice. Nat Commun 9:4129.

Liu Y, Zhou LJ, Wang J, Li D, Ren WJ, Peng J, Wei X, Xu T, Xin WJ, Pang RP, Li YY, Qin ZH, Murugan M, Mattson MP, Wu LJ, Liu XG (2017) TNF- $\alpha$ differentially regulates synaptic plasticity in the hippocampus and spinal cord by microglia-dependent mechanisms after peripheral nerve injury. J Neurosci 37:871-881.

Lohman AW, Isakson BE (2014) Differentiating connexin hemichannels and pannexin channels in cellular ATP release. FEBS Lett 588:1379-1388.

Lu J, Greco MA, Shiromani P, Saper CB (2000) Effect of lesions of the ventrolateral preoptic nucleus on NREM and REM sleep. J Neurosci 20:38303842.

Mackiewicz M, Nikonova EV, Zimmerman JE, Galante RJ, Zhang L, Cater JR, Geiger JD, Pack AI (2003) Enzymes of adenosine metabolism in the brain: diurnal rhythm and the effect of sleep deprivation. J Neurochem 85:348-357.

McKenna JT, Cordeira JW, Christie MA, Tartar JL, McCoy JG, Lee E, McCarley RW, Strecker RE (2008) Assessing sleepiness in the rat: a multiple sleep latencies test compared to polysomnographic measures of sleepiness. J Sleep Res 17:365-375.

Methippara MM, Kumar S, Alam MN, Szymusiak R, McGinty D (2005) Effects on sleep of microdialysis of adenosine $\mathrm{A} 1$ and $\mathrm{A} 2 \mathrm{a}$ receptor analogs into the lateral preoptic area of rats. Am J Physiol Regul Integr Comp Physiol 289:R1715-R1723. 
Montero TD, Orellana JA (2015) Hemichannels: new pathways for gliotransmitter release. Neuroscience 286:45-59.

Morairty S, Rainnie D, McCarley R, Greene R (2004) Disinhibition of ventrolateral preoptic area sleep-active neurons by adenosine: a new mechanism for sleep promotion. Neuroscience 123:451-457.

Murase K, Ryu PD, Randic M (1989) Excitatory and inhibitory amino acids and peptide-induced responses in acutely isolated rat spinal dorsal horn neurons. Neurosci Lett 103:56-63.

Murillo-Rodriguez E, Blanco-Centurion C, Gerashchenko D, Salin-Pascual RJ, Shiromani PJ (2004) The diurnal rhythm of adenosine levels in the basal forebrain of young and old rats. Neuroscience 123:361-370.

Nam Y, Kim JH, Kim JH, Jha MK, Jung JY, Lee MG, Choi IS, Jang IS, Lim DG, Hwang SH, Cho HJ, Suk K (2016) Reversible induction of pain hypersensitivity following optogenetic stimulation of spinal astrocytes. Cell Rep 17:3049-3061.

Oda K, Vierock J, Oishi S, Rodriguez-Rozada S, Taniguchi R, Yamashita K, Wiegert JS, Nishizawa T, Hegemann P, Nureki O (2018) Crystal structure of the red light-activated channelrhodopsin chrimson. Nat Commun 9:3949.

Panatier A, Vallée J, Haber M, Murai KK, Lacaille JC, Robitaille R (2011) Astrocytes are endogenous regulators of basal transmission at central synapses. Cell 146:785-798.

Pascual O, Casper KB, Kubera C, Zhang J, Revilla-Sanchez R, Sul JY, Takano H, Moss SJ, McCarthy K, Haydon PG (2005) Astrocytic purinergic signaling coordinates synaptic networks. Science 310:113-116.

Pelluru D, Konadhode RR, Bhat NR, Shiromani PJ (2016) Optogenetic stimulation of astrocytes in the posterior hypothalamus increases sleep at night in C57BL/6J mice. Eur J Neurosci 43:1298-1306.

Perea G, Araque A (2005) Properties of synaptically evoked astrocyte calcium signal reveal synaptic information processing by astrocytes. J Neurosci 25:2192-2203.

Perea G, Sur M, Araque A (2014) Neuron-glia networks: integral gear of brain function. Front Cell Neurosci 8:378.

Porkka-Heiskanen T (2013) Sleep homeostasis. Curr Opin Neurobiol 23:799-805

Porkka-Heiskanen T, Kalinchuk AV (2011) Adenosine, energy metabolism and sleep homeostasis. Sleep Med Rev 15:123-135.

Porkka-Heiskanen T, Strecker RE, Thakkar M, Bjorkum AA, Greene RW, McCarley RW (1997) Adenosine: a mediator of the sleep-inducing effects of prolonged wakefulness. Science 276:1265-1268.

Porkka-Heiskanen T, Strecker RE, McCarley RW (2000) Brain site-specificity of extracellular adenosine concentration changes during sleep deprivation and spontaneous sleep: an in vivo microdialysis study. Neuroscience 99:507-517.

Porter JT, McCarthy KD (1996) Hippocampal astrocytes in situ respond to glutamate released from synaptic terminals. J Neurosci 16:5073-5081.

Radulovacki M, Virus RM, Djuricic-Nedelson M, Green RD (1984) Adenosine analogs and sleep in rats. J Pharmacol Exp Ther 228:268-274.

Santello M, Calì C, Bezzi P (2012) Gliotransmission and the tripartite synapse. Adv Exp Med Biol 970:307-331.

Saper CB, Fuller PM, Pedersen NP, Lu J, Scammell TE (2010) Sleep state switching. Neuron 68:1023-1042.

Serrano A, Haddjeri N, Lacaille JC, Robitaille R (2006) GABAergic network activation of glial cells underlies hippocampal heterosynaptic depression. J Neurosci 26:5370-5382.
Sherin JE, Shiromani PJ, McCarley RW, Saper CB (1996) Activation of ventrolateral preoptic neurons during sleep. Science 271:216-219.

Sherin JE, Elmquist JK, Torrealba F, Saper CB (1998) Innervation of histaminergic tuberomammillary neurons by GABAergic and galaninergic neurons in the ventrolateral preoptic nucleus of the rat. J Neurosci 18:47054721.

Steininger TL, Gong H, McGinty D, Szymusiak R (2001) Subregional organization of preoptic area/anterior hypothalamic projections to arousalrelated monoaminergic cell groups. J Comp Neurol 429:638-653.

Strecker RE, Morairty S, Thakkar MM, Porkka-Heiskanen T, Basheer R, Dauphin LJ, Rainnie DG, Portas CM, Greene RW, McCarley RW (2000) Adenosinergic modulation of basal forebrain and preoptic/anterior hypothalamic neuronal activity in the control of behavioral state. Behav Brain Res 115:183-204

Street SE, Kramer NJ, Walsh PL, Taylor-Blake B, Yadav MC, King IF, Vihko P, Wightman RM, Millán JL, Zylka MJ (2013) Tissue-nonspecific alkaline phosphatase acts redundantly with PAP and NT5E to generate adenosine in the dorsal spinal cord. J Neurosci 33:11314-11322.

Suadicani SO, Brosnan CF, Scemes E (2006) P2X7 receptors mediate ATP release and amplification of astrocytic intercellular $\mathrm{Ca} 2+$ signaling. J Neurosci 26:1378-1385

Suzuki T, Hide I, Ido K, Kohsaka S, Inoue K, Nakata Y (2004) Production and release of neuroprotective tumor necrosis factor by $\mathrm{P} 2 \mathrm{X} 7$ receptoractivated microglia. J Neurosci 24:1-7.

Szymusiak R, Alam N, Steininger TL, McGinty D (1998) Sleep-waking discharge patterns of ventrolateral preoptic/anterior hypothalamic neurons in rats. Brain Res 803:178-188.

Ticho SR, Radulovacki M (1991) Role of adenosine in sleep and temperature regulation in the preoptic area of rats. Pharmacol Biochem Behav 40:3340.

Timofeev I, Chauvette S (2017) Sleep slow oscillation and plasticity. Curr Opin Neurobiol 44:116-126.

Tobler I, Borbély AA (1990) The effect of 3-h and 6-h sleep deprivation on sleep and EEG spectra of the rat. Behav Brain Res 36:73-78.

Van Poucke M, Martlé V, Van Brantegem L, Ducatelle R, Van Ham L, Bhatti S, Peelman LJ (2016) A canine orthologue of the human GFAP c.716G $>$ A (p.Arg239His) variant causes Alexander disease in a Labrador retriever. Eur J Hum Genet 24:852-856.

Wang X, Lou N, Xu Q, Tian GF, Peng WG, Han X, Kang J, Takano T, Nedergaard M (2006) Astrocytic Ca2 + signaling evoked by sensory stimulation in vivo. Nat Neurosci 9:816-823.

Xing L, Yang T, Cui S, Chen G (2019) Connexin hemichannels in astrocytes: role in CNS disorders. Front Mol Neurosci 12:23.

Xiong Y, Teng S, Zheng L, Sun S, Li J, Guo N, Li M, Wang L, Zhu F, Wang C, Rao Z, Zhou Z (2018) Stretch-induced $\mathrm{Ca}(2+)$ independent ATP release in hippocampal astrocytes. J Physiol 596:1931-1947.

Zhang J, Yin D, Wu F, Zhang G, Jiang C, Li Z, Wang L, Wang K (2013) Microinjection of adenosine into the hypothalamic ventrolateral preoptic area enhances wakefulness via the A1 receptor in rats. Neurochem Res 38:1616-1623.

Zimmermann H (2000) Extracellular metabolism of ATP and other nucleotides. Naunyn Schmiedebergs Arch Pharmacol 362:299-309.

Zylka MJ, Sowa NA, Taylor-Blake B, Twomey MA, Herrala A, Voikar V, Vihko P (2008) Prostatic acid phosphatase is an ectonucleotidase and suppresses pain by generating adenosine. Neuron 60:111-122. 